\title{
Quilted Floer trajectories with constant components: Corrigendum to the article "Quilted Floer cohomology"
}

\author{
KATRIN WEHRHEIM \\ CHRIS T WOODWARD
}

\begin{abstract}
We fill a gap in the proof of the transversality result for quilted Floer trajectories in [10] by addressing trajectories for which some but not all components are constant. Namely we show that for generic sets of split Hamiltonian perturbations and split almost complex structures, the moduli spaces of parametrized quilted Floer trajectories of a given index are smooth of expected dimension. An additional benefit of the generic split Hamiltonian perturbations is that they perturb the given cyclic Lagrangian correspondence such that any geometric composition of its factors is transverse and hence immersed.
\end{abstract}

53D40; 57R56

\section{Introduction}

Quilted Floer homology is defined in [10] for a cyclic generalized Lagrangian correspondence $\underline{L}$, that is, a sequence of symplectic manifolds $M_{0}, M_{1}, \ldots, M_{r}, M_{r+1}$ with $M_{0}=M_{r+1}$ for some $r \geq 0$, and a sequence of compact Lagrangian correspondences

$$
L_{01} \subset M_{0}^{-} \times M_{1}, L_{12} \subset M_{1}^{-} \times M_{2}, \ldots, L_{r(r+1)} \subset M_{r}^{-} \times M_{r+1} .
$$

In [10] we moreover make monotonicity, grading, and Maslov index assumptions that guarantee compactification properties. These are not required for the results in this paper. Quilted Floer homology $H F(\underline{L})$ can be defined as the standard Floer homology of a pair of Lagrangians in the product manifold $M_{0}^{-} \times M_{1} \times M_{2}^{-} \times \cdots \times M_{r}$, given by products of the $L_{i(i+1)}$. (For even $r$ one adds a diagonal to the sequence before making this construction.) As such, the differential counts moduli spaces of pseudoholomorphic strips with Lagrangian boundary conditions for a choice of a Hamiltonian perturbation and almost complex structure on this product manifold so that the Cauchy-Riemann operator cuts out the space of pseudoholomorphic maps transversely. Generically this choice would not be of split form, ie induced by a tuple of Hamiltonian functions and almost complex structures on each symplectic manifold $M_{j}$. The quilted definition of $H F(\underline{L})$ in [10] on the one hand generalizes this construction 
by allowing a choice of widths $\underline{\delta}=\left(\delta_{j}>0\right)_{j=0, \ldots, r}$ of the strips mapping to each $M_{j}$. On the other hand, we claim in [10] that the quilted Floer complex can be constructed (in particular transversality of the quilted Cauchy-Riemann operator can be achieved) for Hamiltonians and almost complex structures of split type. That is, we restrict our choice of perturbation data to a tuple of Hamiltonian functions and a tuple of almost complex structures in the complete metric spaces

$$
\mathcal{H}_{t}(\underline{\delta}):=\bigoplus_{j=0}^{r} \mathcal{C}^{\infty}\left(\left[0, \delta_{j}\right] \times M_{j}, \mathbb{R}\right), \quad \mathcal{J}_{t}(\underline{\delta}):=\bigoplus_{j=0}^{r} \mathcal{C}^{\infty}\left(\left[0, \delta_{j}\right], \mathcal{J}\left(M_{j}, \omega_{j}\right)\right),
$$

where $\mathcal{J}\left(M_{j}, \omega_{j}\right)$ is the space of smooth $\omega_{j}$-compatible almost complex structures on $M_{j}$. (The latter inherits a complete metric as a closed subset of the complete metric vector space $\mathcal{C}^{\infty}\left(\left[0, \delta_{j}\right] \times M_{j}, \operatorname{End}\left(T M_{j}\right)\right)$.) While this split form is not necessary for our strip-shrinking analysis in [11], it is particularly helpful for constructing relative invariants (such as the functor associated to a correspondence in our article [9]) from more complicated quilted surfaces, which cannot be interpreted as single surface mapping to a product manifold. Unfortunately, the transversality proof in [10] for the quilted Floer trajectory spaces for generic split perturbation data $\underline{H} \in \mathcal{H}_{t}(\underline{\delta})$ and $\underline{J} \in \mathcal{J}_{t}(\underline{\delta})$ has a significant gap: It fails to explicitly discuss trajectories $\underline{u}=$ $\left(u_{j}: \mathbb{R} \times\left[0, \delta_{j}\right] \rightarrow M_{j}\right)_{j=0, \ldots, r}$ for which some but not all components are constant. This intermediate situation is not an easy combination of the two extreme cases (all components nonconstant, or all components constant) as we seem to claim in [10].

\section{Results}

In Theorem 3.2 below we complete the proof of the transversality claimed in [10] by working with a more specific set of split Hamiltonian perturbations which may be of independent interest. In Theorem 2.3 and Corollary 2.4 we find a dense open subset of $\mathcal{H}_{t}(\underline{\delta})$ for any given cyclic Lagrangian correspondence such that, after perturbation by one of those split Hamiltonian diffeomorphisms, any geometric composition of its factors is transverse and hence immersed. Starting from such a Hamiltonian perturbation, we observe that quilted Floer trajectories with constant components induce quilted Floer trajectories for a shorter cyclic generalized Lagrangian correspondence, given by a localized version of geometric composition across the constant strips. Using this point of view and a further technical restriction on the Hamiltonian perturbations (see Theorem 2.9(b)), we are able to find generic sets of split almost complex structures for which quilted Floer trajectories with constant components are regular, as well. In fact, we show that quilted Floer trajectories with constant components are very rare as summarized in Remark 3.3 and sketched below.

Note that the technical restriction on the Hamiltonians does not harm the applicability to dynamics, since quilted Floer homology is always equivalent to a standard Floer 
homology of product Lagrangians. The latter is well defined for a given Hamiltonian perturbation yielding transverse Lagrangians and generic (nonsplit) almost complex structure. On the other hand, the same Floer homology can be calculated in the quilted setting, using eg the strip-shrinking isomorphism of [11] or relative invariants defined in [12].

\section{Idea of Proof}

A key role in the proof is played by certain families of isotropic subspaces which arise in the proof of transversality for the universal moduli space of almost complex structures and Floer trajectories. The elements of the cokernel of the linearized operator of the universal moduli space are tuples of $\left(-J_{i}\right)$-holomorphic sections $\eta_{i}$ of $u_{i}^{*} T M_{i}$ for $i=0, \ldots, r$ with Lagrangian seam conditions determined by the tangent spaces of $L_{i(i+1)}$. Ignoring Hamiltonian perturbations for simplicity, the problem of constant components occurs for example when some $u_{j}$ is constant (with value say $x_{j} \in M_{j}$ ) but the adjacent components $u_{j-1}, u_{j+1}$ are nonconstant. Then variations in the almost complex structures prove vanishing of $\eta_{j-1}, \eta_{j+1}$, and hence $\eta_{j}: \mathbb{R} \times\left[0, \delta_{j}\right] \rightarrow T_{x_{j}} M_{j}$ is $\left(-J_{j}\right)$-holomorphic with boundary conditions in

$$
\begin{aligned}
& \Lambda_{j}(s):=\Pi_{T_{x_{j}} M_{j}}\left(T_{\left(u_{j-1}\left(\delta_{j-1}, s\right), x_{j}\right)} L_{(j-1) j} \cap\left(\{0\} \times T_{x_{j}} M_{j}\right)\right) \subset T_{x_{j}} M_{j}, \\
& \Lambda_{j}^{\prime}(s):=\Pi_{T_{x_{j}} M_{j}}\left(T_{\left(x_{j}, u_{j+1}(0, s)\right)} L_{j(j+1)} \cap\left(T_{x_{j}} M_{j} \times\{0\}\right)\right) \quad \subset T_{x_{j}} M_{j},
\end{aligned}
$$

where $\Pi_{T_{x_{j}} M_{j}}$ denotes the projection onto $T_{x_{j}} M_{j}$ (an injection in both cases). The spaces $\Lambda_{j}(s), \Lambda_{j}^{\prime}(s)$ are isotropic spaces varying with $s \in \mathbb{R}$, despite the fact that $u_{j} \equiv x_{j}$ is constant. We can now proceed differently in three nonexclusive cases.

(a) The easiest case is to assume that $\Lambda_{j}(s), \Lambda_{j}^{\prime}(s)$ are $s$-independent. We may then enlarge these isotropic spaces to constant Lagrangian subspaces and deduce that $\eta_{j}$ lies in the kernel of an operator $\partial_{s}+A$, where $A$ is an $s$-independent self-adjoint operator and invertible (since by choice of $\underline{H}$ the generators of the Floer complex are cut out transversally). We then deduce vanishing of $\eta_{j}$ from the general fact that operators of this form $\partial_{s}+A$ are isomorphisms.

(b) An intermediate case occurs when $\Lambda_{j}(s)$ or $\Lambda_{j}^{\prime}(s)$ fails to be Lagrangian (ie have maximal dimension) for some $s \in \mathbb{R}$. For example, if $L_{(j-1) j}$ is the graph of a symplectomorphism, then the intersection $\Lambda_{j}$ is trivial. We show that this case of a quilted Floer trajectory with constant component does not occur for generic $\left(J_{i}\right)_{i \neq j}$.

(c) The most difficult case occurs when $\Lambda_{j}(s)$ and $\Lambda_{j}^{\prime}(s)$ are nonconstant families of Lagrangian subspaces. We show that for generic $\underline{H}$ the locus on which such varying Lagrangian subspaces are possible is of positive codimension in the 
space of boundary values $\left(u_{j-1}\left(\delta_{j-1}, s\right), u_{j+1}(0, s)\right)$. Then we again exclude this case for generic $\left(J_{i}\right)_{i \neq j}$.

Thus, for generic Hamiltonian perturbations $\underline{H}$ and almost complex structures $\underline{J}$ we in fact show a splitting property for any quilted Floer trajectory with constant components, namely along the seam $\left(u_{j}\left(s, \delta_{j}\right), u_{j+1}(s, 0)\right) \in L_{j(j+1)}$ we have $T L_{j(j+1)}=$ $\Lambda_{j} \times \Lambda_{j+1}$, where $\Lambda_{j} \subset T M_{j}$ is a constant Lagrangian subspace given as above, and $\Lambda_{j+1}$ is the $s$-dependent projection of $T L_{j(j+1)} \cap\left(\{0\} \times T M_{j+1}\right)$. For a precise statement see Remark 3.3.

The arguments in case (b) and (c) crucially rely on the following interpretation of quilted Floer trajectories with constant components as quilted Floer trajectories for a generalized Lagrangian correspondence obtained by a local version of geometric composition. If $\underline{u}=\left(u_{0}, \ldots, u_{r}\right)$ is a solution with $u_{j} \equiv x_{j}$ as above, then $\left(u_{0}, \ldots, u_{j-1}, u_{j+1}, \ldots, u_{r}\right)$ is a quilted Floer trajectory for the generalized correspondence $\left(L_{01}, \ldots, L_{(j-1) j} \circ L_{j(j+1)}, \ldots, L_{r(r+1)}\right)$. We show in Theorem 2.3 that, after a generic Hamiltonian perturbation of $\underline{L}$, any geometric composition $L_{(j-1) j} \circ L_{j(j+1)}$ is an immersed Lagrangian correspondence. It becomes embedded if we restrict to values in $M_{j}$ near $x_{j}$. Hence $\left(u_{0}, \ldots, u_{j-1}, u_{j+1}, \ldots, u_{r}\right)$ can be viewed as quilted Floer trajectory for a smooth generalized Lagrangian correspondence.

We showed in [11] that transversality for this composed correspondence implies transversality for the original correspondence for sufficiently small widths $\delta_{j}>0$. Here we extend this transversality to solutions with constant $u_{j}$ for arbitrary $\delta_{j}>0$ and generic perturbation data $\underline{H}, \underline{J}$.

\section{Alternative approaches}

It is perhaps worth remarking that all of the correspondences intended as applications in $[10 ; 14 ; 13]$ fit into the easiest case (a) described above since these Lagrangian correspondences $L_{01} \subset M_{0}^{-} \times M_{1}$ are quasisplit in the following sense: The intersection $\left(T_{x_{0}} M_{0} \times\{0\}\right) \cap T_{\left(x_{0}, x_{1}\right)} L_{01}$ is independent of $x_{1}$ and the intersection $\left(\{0\} \times T_{x_{1}} M_{1}\right) \cap T_{\left(x_{0}, x_{1}\right)} L_{01}$ is independent of $x_{0}$. Examples are split correspondences $L_{0} \times L_{1}$, graphs of symplectomorphisms, correspondences arising from fibered coisotropics, and the embedded geometric composition of any two quasisplit correspondences. If all Lagrangian correspondences are quasisplit then the simple argument in case (a) above completes the transversality argument for the universal moduli space in [10].

Note however that one can easily construct Lagrangian correspondences that are not quasisplit by applying a nonsplit Hamiltonian diffeomorphism of $M_{0}^{-} \times M_{1}$ to a split correspondence $L_{0} \times L_{1}$. 
Another possibility for achieving transversality at quilted Floer trajectories with constant components is to introduce nonsplit perturbations as in Perutz [5]. However, in order to implement this perturbation scheme for more general relative quilt invariants, one would have to replace each seam with seam condition $L_{i j} \subset M_{i} \times M_{j}$ by a strip in $M_{i} \times M_{j}$, one of whose boundaries takes values in $L_{i j}$, and the other boundary gets paired via a diagonal with the two adjacent patches in $M_{i}$ and $M_{j}$ - a novel type of seam condition, relating three patches at one common boundary component. In that setup we may use nonsplit perturbations on the strip.

Acknowledgement We thank Maksim Lipyanskiy for pointing out the question of constant components, and the referee for substantial help in improving the exposition.

\section{Hamiltonian perturbations of generalized Lagrangian cor- respondences}

Given a cyclic generalized Lagrangian correspondence $\underline{L}=\left(L_{j(j+1)}\right)_{j=0, \ldots, r}$, widths $\underline{\delta}=\left(\delta_{j}>0\right)_{j=0, \ldots, r}$, and a tuple of Hamiltonian functions $\underline{H}=\left(H_{j}\right)_{j=0, \ldots, r} \in \mathcal{H}_{t}(\underline{\delta})$, the generators of the quilted Floer complex are tuples of Hamiltonian chords,

$$
\mathcal{I}(\underline{L}, \underline{H}):=\left\{\begin{array}{l|l}
\underline{x}=\left(x_{j}:\left[0, \delta_{j}\right] \rightarrow M_{j}\right)_{j=0, \ldots, r} & \begin{array}{l}
\dot{x}_{j}(t)=X_{H_{j}}\left(x_{j}(t)\right), \\
\left(x_{j}\left(\delta_{j}\right), x_{j+1}(0)\right) \in L_{j(j+1)}
\end{array}
\end{array}\right\} .
$$

They are canonically identified, via $\underline{x} \mapsto\left(x_{0}\left(\delta_{0}\right), x_{1}(0), x_{1}\left(\delta_{1}\right), \ldots, x_{r}\left(\delta_{r}\right), x_{0}(0)\right)$, with the fiber product

$$
\begin{aligned}
& \times_{\phi_{\delta_{0}}^{H_{0}}}\left(L_{01} \times_{\phi_{\delta_{1}}^{H_{1}}} L_{12} \cdots \times{ }_{\phi_{\delta_{r}}^{H_{r}}} L_{r(r+1)}\right) \\
& :=\left(L_{01} \times L_{12} \times \cdots \times L_{r(r+1)}\right) \cap\left(\operatorname{graph}\left(\phi_{\delta_{1}}^{H_{1}}\right) \times \operatorname{graph}\left(\phi_{\delta_{2}}^{H_{2}}\right) \times \cdots \times \operatorname{graph}\left(\phi_{\delta_{0}}^{H_{0}}\right)\right)^{T},
\end{aligned}
$$

where $\phi_{\delta_{j}}^{H_{j}}$ is the time $\delta_{j}$ Hamiltonian flow of $H_{j}$ and $(\cdots)^{T}$ denotes the exchange of factors $M_{1} \times \cdots \times M_{0} \times M_{0} \rightarrow M_{0} \times M_{1} \times \cdots \times M_{0}$. In this setting we proved in [10] that Hamiltonians of split type suffice to achieve transversality for the generators. We now strengthen this result to achieve transversality for all partial fiber products.

Convention 2.1 Here and in the following the indices are mostly used modulo $r+1$ but still denoted by $j \in \mathbb{N}_{0}$ in order to have the following notation: A pair of indices $j<j^{\prime}$ denotes a pair $j, j^{\prime} \in \mathbb{N}_{0}$ with $j<j^{\prime} \leq j+r+1$. A pair of indices $j \triangleleft j^{\prime}$ denotes a pair $j, j^{\prime} \in \mathbb{N}_{0}$ with $j+1<j^{\prime} \leq j+r+1$, that is, with at least one other index between $j$ and $j^{\prime}$. 
For any proper subset $I \subset\{0, \ldots, r\}$ let $I^{C} \subset\{0, \ldots, r\}$ be its complement. Then a consecutive pair of indices $j<j^{\prime} \in I^{C}$ (resp. $j \triangleleft j^{\prime} \in I^{C}$ ) denotes a pair $j<j^{\prime}$ (resp. $j \triangleleft j^{\prime}$ ) as above such that $j, j^{\prime} \in I^{C}$ and $\left\{j+1, \ldots, j^{\prime}-1\right\} \subset I$.

Definition 2.2 For any pair of indices $j \triangleleft j^{\prime}$ we define the partial fiber product

$$
\begin{aligned}
& L_{j(j+1)} \times{ }_{H_{j+1}} L_{(j+1)(j+2)} \cdots \times{ }_{H_{j^{\prime}-1}} L_{\left(j^{\prime}-1\right) j^{\prime}} \\
& :=\left(L_{j(j+1)} \times \cdots \times L_{\left(j^{\prime}-1\right) j^{\prime}}\right) \cap\left(M_{j} \times \operatorname{graph}\left(\phi_{\delta_{j+1}}^{H_{j+1}}\right) \times \cdots \times \operatorname{graph}\left(\phi_{\delta_{j^{\prime}-1}}^{H_{j^{\prime}-1}}\right) \times M_{j^{\prime}}\right) .
\end{aligned}
$$

We trivially extend this notation to the case $j^{\prime}=j+1$ by $L_{j j^{\prime}}=L_{j(j+1)}$. For a general proper subset of indices $I \subset\{0, \ldots, r\}$ we then define the partial fiber product

$$
\times_{I, \underline{H}} \underline{L}:=\prod_{\text {consec. } j<j^{\prime} \in I^{C}} L_{j(j+1)} \times_{H_{j+1}} \cdots \times_{H_{j^{\prime}-1}} L_{\left(j^{\prime}-1\right) j^{\prime}}
$$

to be the product of the above fiber products for each consecutive pair of indices $j<j^{\prime} \in I^{C}$. We view the intersection $\mathcal{I}(\underline{L}, \underline{H})=\times_{\{0, \ldots, r\}, \underline{H}} \underline{L}$ as the full fiber product case $I=\{0, \ldots, r\}$.

Given $\underline{L}$, we call a tuple of Hamiltonian functions $\underline{H} \in \mathcal{H}_{t}(\underline{\delta})$ quilted regular if the defining equations for $\times_{I, \underline{H}} \underline{L}$ for any $I \subset\{0, \ldots, r\}$ are transversal.

Theorem 2.3 The set $\operatorname{Ham}^{*}(\underline{L}) \subset \mathcal{H}_{t}(\underline{\delta})$ of quilted regular tuples of Hamiltonians is a dense open subset of $\mathcal{H}_{t}(\underline{\delta})$.

Proof Each of the fiber products under consideration is of the following form: It is the set of tuples $\left(m_{0}^{\prime}, m_{1}, m_{1}^{\prime}, \ldots, m_{r}^{\prime}, m_{r+1}\right) \in L_{01} \times \cdots \times L_{r(r+1)}$ satisfying

$$
\phi_{\delta_{i}}^{H_{i}}\left(m_{i}\right)=m_{i}^{\prime} \quad \forall i \in I .
$$

It suffices to show that the subset of regular Hamiltonians for each of these problems is dense and open, since the intersection of finitely many dense open subsets remains dense and open. So we fix some choice of $I \subset\{0, \ldots, r\}$ and consider the universal moduli $\mathcal{M}^{\text {univ }}$ space of data $\left(H_{0}, \ldots, H_{r}, m_{0}^{\prime}, m_{1}, \ldots, m_{r}^{\prime}, m_{r+1}\right)$ satisfying (1), where now each $H_{j}$ has class $\mathcal{C}^{\ell+1}$ for some $\ell>\sum_{i \in I^{C}} \operatorname{dim} M_{i}$. It is cut out by the diagonal values of the $\mathcal{C}^{\ell}$-map

$$
\begin{aligned}
L_{01} \times L_{12} \cdots \times L_{r(r+1)} \times \bigoplus_{k=0}^{r} \mathcal{C}^{\ell+1}\left(\left[0, \delta_{k}\right] \times M_{k}\right) & \longrightarrow \prod_{j \in I} M_{j} \times M_{j}, \\
\left(m_{0}^{\prime}, m_{1}, m_{1}^{\prime}, m_{2}, \ldots, m_{r}^{\prime}, m_{r+1}, H_{0}, \ldots, H_{r}\right) & \longmapsto\left(\phi_{\delta_{i}}^{H_{i}}\left(m_{i}\right), m_{i}^{\prime}\right)_{i \in I} .
\end{aligned}
$$


The linearized equations for $\mathcal{M}^{\text {univ }}$ are

$$
v_{i}^{\prime}-D \phi_{\delta_{i}}^{H_{i}}\left(h_{i}, v_{i}\right)=0 \in T M_{i} \quad \forall i \in I .
$$

for $v_{i} \in T_{m_{i}} M_{i}, v_{i}^{\prime} \in T_{m_{i}^{\prime}} M_{i}$, and $h_{i} \in \mathcal{C}^{\ell+1}\left(\left[0, \delta_{i}\right] \times M_{i}\right)$. The map

$$
\mathcal{C}^{\ell+1}\left(\left[0, \delta_{i}\right] \times M_{i}\right) \rightarrow T_{\phi_{\delta_{i}}^{H_{i}}\left(m_{i}\right)} M_{i}, \quad h_{i} \mapsto D \phi_{\delta_{i}}^{H_{i}}\left(h_{i}, 0\right)
$$

is surjective, which shows that the product of the operators on the left-hand side of (2) is also surjective. So by the implicit function theorem $\mathcal{M}^{\text {univ }}$ is a $\mathcal{C}^{\ell}$ Banach manifold, and we consider its projection to $\bigoplus_{k=0}^{r} \mathcal{C}^{\ell+1}\left(\left[0, \delta_{k}\right] \times M_{k}\right)$. This is a Fredholm map of class $\mathcal{C}^{\ell}$ and index $\sum_{i \in I^{C}} \operatorname{dim} M_{i}$ (in particular 0 for the full intersection $I=\{0, \ldots, r\})$. Indeed, the Fredholm property and index follow eg from McDuff and Salamon [4, A.3.6]); differentiability follows from being a restriction of the smooth projection

$$
L_{01} \times \ldots L_{r(r+1)} \times \bigoplus_{k=0}^{r} \mathcal{C}^{\ell+1}\left(\left[0, \delta_{k}\right] \times M_{k}\right) \rightarrow \bigoplus_{k=0}^{r} \mathcal{C}^{\ell+1}\left(\left[0, \delta_{k}\right] \times M_{k}\right)
$$

to a $\mathcal{C}^{\ell}$ Banach submanifold. Hence, by the Sard-Smale theorem the set of regular values (which coincides with the set of functions $\underline{H}=\left(H_{0}, \ldots, H_{r}\right)$ such that the perturbed intersection is transversal) is dense in $\bigoplus_{k=0}^{r} \mathcal{C}^{\ell+1}\left(\left[0, \delta_{k}\right] \times M_{k}\right)$. Moreover, the set of regular values is open for each $\ell>\sum_{i \in I^{C}} \operatorname{dim} M_{i}$. Indeed, by the compactness of $L_{01} \times L_{12} \cdots \times L_{r(r+1)}$, a $\mathcal{C}^{1}$-small change in $\underline{H}$ leads to a small change in perturbed intersection points, with small change in the linearized operators.

Now, by approximation of $\mathcal{C}^{\infty}$-functions with $\mathcal{C}^{\ell+1}$-functions, the set of regular values in $\bigoplus_{k=0}^{r} \mathcal{C}^{\infty}\left(\left[0, \delta_{k}\right] \times M_{k}\right)$ is dense in the $\mathcal{C}^{\ell+1}$-topology for all $\ell>\sum_{i \in I^{C}} \operatorname{dim} M_{i}$, and hence dense in the $\mathcal{C}^{\infty}$-topology. Finally, the set of regular smooth $\underline{H}$ is open in the $\mathcal{C}^{\infty}$-topology as a special case of the $\mathcal{C}^{1}$-openness.

We now reformulate this Theorem by using the Hamiltonian flows of $\underline{H}$ to perturb the Lagrangian correspondences and then applying a geometric composition in some factors.

Corollary 2.4 For $\underline{H} \in \operatorname{Ham}^{*}(\underline{L})$ the perturbed generalized correspondence

$$
\underline{L}^{\prime}:=\left(L^{\prime}:=\left(\operatorname{Id}_{\mathrm{M}_{\mathrm{j}}} \times \phi_{\delta_{j+1}}^{H_{j+1}}\right) L_{j(j+1)}\right)_{j=0, \ldots, r}
$$

has the following intersection and composition properties:

(a) The generalized intersection

$$
\mathcal{I}\left(\underline{L}^{\prime}, 0\right)=\left(L_{01}^{\prime} \times \cdots \times L_{r(r+1)}^{\prime}\right) \cap\left(\Delta_{M_{1}} \times \cdots \times \Delta_{M_{0}}\right)^{T}
$$

is transverse and canonically identified with $\mathcal{I}(\underline{L}, \underline{H})$. 
(b) For the perturbed correspondence $\underline{L}^{\prime}$ the trivial Hamiltonian $\underline{0} \in \mathcal{H}_{t}(\delta)$ is quilted regular. That is, for any proper subset $I \subset\{0, \ldots, r\}$ the partial fiber product $\times_{I, 0} \underline{L}^{\prime}$ is cut out transversally (and canonically identified with $\times_{I, \underline{H}} \underline{L}$ ). It is a product of the transverse intersections

$$
\begin{aligned}
& L_{j(j+1)}^{\prime} \times_{\Delta_{j+1}} L_{(j+1)(j+2)}^{\prime} \times_{\Delta_{j+2}} \cdots \times_{\Delta_{j^{\prime}-1}} L_{\left(j^{\prime}-1\right) j^{\prime}}^{\prime} \\
& :=\left(L_{j(j+1)}^{\prime} \times L_{(j+1)(j+2)}^{\prime} \times \cdots \times L_{\left(j^{\prime}-1\right) j^{\prime}}^{\prime}\right) \cap\left(M_{j} \times \Delta_{M_{j+1}} \cdots \Delta_{M_{j^{\prime}-1}} \times M_{j^{\prime}}\right) \\
& =\left(\operatorname{Id}_{M_{j}} \times \phi_{\delta_{j+1}}^{H_{j+1}} \times \operatorname{Id}_{M_{j+1}} \times \cdots \times \phi_{\delta_{j^{\prime}-1}}^{H_{j^{\prime}-1}} \times \phi_{\delta_{j^{\prime}}}^{H_{j^{\prime}}}\right)\left(L_{j(j+1)} \times{ }_{H_{j+1}} \cdots \times \times_{H_{j^{\prime}-1}} L_{\left(j^{\prime}-1\right) j^{\prime}}\right)
\end{aligned}
$$

for consecutive pairs of indices $j<j^{\prime} \in I^{C}$.

(c) By a direct generalization of [10, Lemma 2.0.5], the projection

$$
\Pi_{M_{j} \times M_{j^{\prime}}}: L_{j(j+1)}^{\prime} \times_{\Delta_{j+1}} \cdots \times_{\Delta_{j^{\prime}-1}} L_{\left(j^{\prime}-1\right) j^{\prime}}^{\prime} \longrightarrow M_{j} \times M_{j^{\prime}}
$$

is an immersion onto the geometric composition

$$
L_{j(j+1)}^{\prime} \circ \ldots \circ L_{\left(j^{\prime}-1\right) j^{\prime}}^{\prime} \subset M_{j} \times M_{j^{\prime}} .
$$

We will in particular be interested in the geometric composition near a fixed point in $M_{j+1} \times \cdots \times M_{j^{\prime}-1}$ given by the components of an intersection point in $\mathcal{I}\left(\underline{L}^{\prime}, 0\right)$. For any such point there is a neighborhood $\mathcal{U} \subset M_{j+1} \times \cdots \times M_{j^{\prime}-1}$ such that the projection $\Pi_{M_{j} \times M_{j^{\prime}}}$ embeds $\left(L_{j(j+1)}^{\prime} \times_{\Delta_{j+1}} L_{(j+1)(j+2)}^{\prime} \cdots \times \times_{\Delta_{j^{\prime}-1}} L_{\left(j^{\prime}-1\right) j^{\prime}}^{\prime}\right) \cap\left(M_{j} \times \mathcal{U} \times M_{j^{\prime}}\right)$ into $M_{j} \times M_{j^{\prime}}$. This is a localized version of the embedded geometric composition (as studied in [10]) of the perturbed Lagrangians. We will be using the following analogue of the perturbed geometric composition of unperturbed Lagrangian correspondences.

Definition 2.5 Let $\underline{H} \in \operatorname{Ham}^{*}(\underline{L})$ be quilted regular. Then for a proper subset $I \subset\{0, \ldots, r\}$ and $\underline{x} \in \mathcal{I}(\underline{L}, \underline{H})$ we define the locally composed cyclic Lagrangian correspondence $\underline{L}, \underline{\underline{H}}, \underline{x}$ between the underlying manifolds $\left(M_{j}\right)_{j \in I^{C}}$ to be the cyclic sequence consisting of $L_{j j^{\prime}}^{\underline{H}} \subset M_{j} \times M_{j^{\prime}}$ for each consecutive pair of indices $j<j^{\prime} \in I^{C}$, given by

$$
L_{j j^{\prime}}^{\underline{H}, \underline{x}}:=\Pi_{M_{j} \times M_{j^{\prime}}}\left(\left(L_{j(j+1)} \times_{H_{j+1}} L_{(j+1)(j+2)} \cdots \times_{H_{j^{\prime}-1}} L_{\left(j^{\prime}-1\right) j^{\prime}}\right) \cap \tilde{\mathcal{U}}_{\underline{x}, j, j^{\prime}}\right)
$$

for $\tilde{\mathcal{U}}_{\underline{x}, j, j^{\prime}}:=M_{j} \times \mathcal{U}_{\underline{x}, j_{, j}} \times M_{j^{\prime}}$, where $\mathcal{U}_{\underline{x}, j, j^{\prime}}$ is a chosen neighborhood of the point $\left(x_{j+1}(0), x_{j+1}\left(\delta_{j+1}\right), \ldots, x_{j^{\prime}-1}(0), x_{j^{\prime}-1}\left(\delta_{j^{\prime}-1}\right)\right)$ such that $\Pi_{M_{j} \times M_{j^{\prime}}}$ is injective on the intersection.

Remark 2.6 Given a regular tuple of Hamiltonian functions $\underline{H} \in \operatorname{Ham}^{*}(\underline{L})$ as in Theorem 2.3 and a proper subset $I \subset\{0, \ldots, r\}$, let $\underline{\delta}^{I}:=\left(\delta_{j}\right)_{j \in I^{C}}$ and $\underline{H}^{I}:=$ $\left(H_{j}\right)_{j \in I^{C}}$. Then the transversality assertions of Theorem 2.3 moreover imply that for 
any $\underline{x} \in \mathcal{I}(\underline{L}, \underline{H})$ the intersection $\mathcal{I}\left(\underline{L} \underline{L}^{I, \underline{H}}, \underline{x}, \underline{H}^{I}\right)$ is transverse. It contains $\left(x_{j}\right)_{j \in I^{C}}$, and no other points if the neighborhoods $\mathcal{U}_{\underline{x}, j, j^{\prime}}$ are chosen sufficiently small.

In preparation for the analysis of quilted Floer trajectories with constant components, we next study the lift from $L \frac{H}{j j^{\prime}} \underline{\underline{x}}$ to $M_{j+1} \times M_{j^{\prime}-1}$ and its connection with the intersections $T L_{j(j+1)} \cap\left(\{0\} \times T M_{j+1}\right)$ and $T L_{\left(j^{\prime}-1\right) j^{\prime}} \cap\left(T M_{j^{\prime}-1} \times\{0\}\right)$. A priori, the latter are isomorphic to collections of isotropic subspaces of $T M_{j+1}$ resp. $T M_{j^{\prime}-1}$ parametrized by $L_{j(j+1)}$ resp. $L_{\left(j^{\prime}-1\right) j^{\prime}}$. As mentioned in the introduction, a first step is to understand the locus where these subspaces are Lagrangian, and how they may vary along $L \frac{H}{j} j^{\prime}, \underline{x}$. For that purpose we introduce the following notation.

Definition 2.7 Let $j \triangleleft j^{\prime}$ be a pair of indices.

(a) Denote by $\mathcal{S}_{j j^{\prime}} \subset L_{j(j+1)} \times L_{\left(j^{\prime}-1\right) j^{\prime}}$ the set of points ${ }^{1} \underline{q}=\left(q_{j}, q_{j+1}, q_{j^{\prime}-1}, q_{j^{\prime}}\right)$ for which

$$
\Lambda_{(j+1)\left(j^{\prime}-1\right)}(\underline{q}):=T_{\underline{q}}\left(L_{j(j+1)} \times L_{\left(j^{\prime}-1\right) j^{\prime}}\right) \cap T_{\underline{q}}\left(\left\{q_{j}\right\} \times M_{j+1} \times M_{j^{\prime}-1} \times\left\{q_{j^{\prime}}\right\}\right)
$$

induces a Lagrangian subspace in $T_{q_{j+1}} M_{j+1} \times T_{q_{j^{\prime}-1}} M_{j^{\prime}-1}$ (with the appropriate signs on the symplectic forms).

(b) Given moreover $\underline{H} \in \operatorname{Ham}^{*}(\underline{L}), \underline{x} \in \mathcal{I}(\underline{L}, \underline{H})$, denote by

$$
\mathcal{P}_{j j^{\prime}}: L_{j j^{\prime}}, \underline{\underline{x}} \longrightarrow M_{j+1} \times M_{j^{\prime}-1}
$$

the composition of the lift from $L_{j j^{\prime}}, \underline{\underline{x}}$ to

$$
\left(L_{j(j+1)} \times_{H_{j+1}} \cdots \times_{H_{j^{\prime}-1}} L_{\left(j^{\prime}-1\right) j^{\prime}}\right) \cap \tilde{\mathcal{U}}_{\underline{x}, j, j^{\prime}}
$$

and the projection to the second and penultimate component, ie to a neighborhood of $\left(x_{j+1}(0), x_{j^{\prime}-1}\left(\delta_{j^{\prime}-1}\right)\right)$.

The following Proposition shows that the set $\mathcal{S}_{j j^{\prime}}$ can equivalently be defined as the locus where the linearized Lagrangian correspondences split, and that this splitting locus is closely related to the vanishing of $D \mathcal{P}_{j j^{\prime}}$.

Proposition 2.8 The following holds for any pair of indices $j \triangleleft j^{\prime}$.

(i) The subset $\mathcal{S}_{j j^{\prime}} \subset L_{j(j+1)} \times L_{\left(j^{\prime}-1\right) j^{\prime}}$ is compact and coincides with the subset for which both $T_{\left(q_{j}, q_{j+1}\right)} L_{j(j+1)}=\Lambda_{j} \times \Lambda_{j+1}$ and $T_{\left(q_{j^{\prime}-1}^{\prime}, q_{j^{\prime}}\right)} L_{\left(j^{\prime}-1\right) j^{\prime}}=$ $\Lambda_{j^{\prime}-1} \times \Lambda_{j^{\prime}}$ are of split form given by Lagrangian subspaces $\Lambda_{i} \subset T_{q_{i}} M_{i}$.

${ }^{1}$ To avoid confusion in the case $j=0 \cong j^{\prime}=r+1$ modulo $r+1$ note that we work with indices in $\mathbb{N}_{0}$ to explicitly allow $q_{j^{\prime}} \neq q_{j}$. 
(ii) For any $\underline{H} \in \operatorname{Ham}^{*}(\underline{L}), \underline{x} \in \mathcal{I}(\underline{L}, \underline{H})$ the linearization

$$
D_{\left(q_{j}, q_{j^{\prime}}\right)} \mathcal{P}_{j j^{\prime}}: T_{\left(q_{j}, q_{j^{\prime}}\right)} L \frac{\underline{H}, \underline{x} j^{\prime}}{\underline{x}} \rightarrow T_{\mathcal{P}_{j j^{\prime}}\left(q_{j}, q_{j^{\prime}}\right)}\left(M_{j+1} \times M_{j^{\prime}-1}\right)
$$

is trivial if and only if $\left(q_{j}, \mathcal{P}_{j j^{\prime}}\left(q_{j}, q_{j^{\prime}}\right), q_{j^{\prime}}\right) \in \mathcal{S}_{j j^{\prime}}$.

Proof We begin by establishing the basic symplectic linear algebra facts that will be used in the proof. Let $V_{1}, V_{2}$ be symplectic vector spaces.

(a) Let $\Lambda_{12} \subset V_{1}^{-} \times V_{2}$ be a linear Lagrangian correspondence. If the intersection $\Lambda_{2}:=\Pi_{V_{2}}\left(\Lambda_{12} \cap\left(\{0\} \times V_{2}\right)\right) \subset V_{2}$ is Lagrangian then $\Lambda_{12}=\Lambda_{1} \times \Lambda_{2}$ splits into $\Lambda_{2}$ and the complementary intersection $\Lambda_{1}:=\Pi_{V_{1}}\left(\Lambda_{12} \cap\left(V_{1} \times\{0\}\right)\right) \subset V_{1}$, which is Lagrangian as well.

(b) In the notation of (a), the intersection $\Lambda_{2}$ is Lagrangian if and only if $\Lambda_{12}=$ $\Lambda_{1}^{\prime} \times \Lambda_{2}^{\prime}$ is the product of two Lagrangian subspaces $\Lambda_{i}^{\prime} \subset V_{i}$.

(c) Let $\mathcal{L}\left(V_{1} \times V_{2}^{-}\right)$be the Lagrangian Grassmannian of $V_{1} \times V_{2}^{-}$and let $\mathcal{S} \subset$ $\mathcal{L}\left(V_{1} \times V_{2}^{-}\right)$be the subset consisting of the Lagrangian subspaces $\Lambda_{12} \subset V_{1}^{-} \times V_{2}$ for which $\Lambda_{2}$ as in (a) is Lagrangian. Then $\mathcal{S}$ is closed.

(d) The same statements hold with $\Lambda_{1}$ and $\Lambda_{2}$ interchanged.

To see (a), note that both $\Lambda_{i} \subset V_{i}$ are automatically isotropic since they are identified with the isotropic subspaces $\Lambda_{1} \times\{0\},\{0\} \times \Lambda_{2} \subset \Lambda_{12}$. Now suppose $\Lambda_{2}$ is Lagrangian, then for any $\left(v_{1}, v_{2}\right) \in \Lambda_{12}$ we have $v_{2} \in \Lambda_{2}^{\omega_{2}}=\Lambda_{2}$, which implies $\left(0, v_{2}\right) \in \Lambda_{12}$, and hence $\left(v_{1}, 0\right) \in \Lambda_{12}$, that is $v_{1} \in \Lambda_{1}$. This shows that $\Lambda_{12}$ splits, and since it is Lagrangian, the first factor $\Lambda_{1}$ must be Lagrangian as well. This proves (a) and one implication of (b); the reverse implication is immediate since $\Lambda_{2}=\Lambda_{2}^{\prime}$. For (c) note that $\{0\} \times V_{2}$ is a symplectic subspace and the projection $\Pi_{V_{2}}:\{0\} \times V_{2} \rightarrow V_{2}$ a symplectic isomorphism. So $\Lambda_{2} \subset V_{2}$ is Lagrangian if and only if the intersection $\Lambda_{12} \cap\left(\{0\} \times V_{2}\right)$ with the symplectic subspace $\{0\} \times V_{2}$ has maximal dimension. This condition is preserved in a limit, so occurs on a closed subset of $\mathcal{L}\left(V_{1} \times V_{2}^{-}\right)$.

Now for a point $\underline{q}=\left(q_{j}, q_{j+1}, q_{j^{\prime}-1}^{\prime}, q_{j^{\prime}}\right) \in L_{j(j+1)} \times L_{\left(j^{\prime}-1\right) j^{\prime}}$ we may apply the above facts to $\Lambda_{12}(\underline{q})=T_{\left(q_{j}, q_{j+1}\right)} L_{j(j+1)}$ and $\Lambda_{12}^{\prime}(\underline{q})=T_{\left(q_{j^{\prime}-1}^{\prime}, q_{j^{\prime}}\right)} L_{\left(j^{\prime}-1\right) j^{\prime}}$ in local trivializations $T_{q_{j}} \bar{M}_{j} \times T_{q_{j+1}} M_{j+1} \cong V_{1} \times V_{2}$ and $\bar{T}_{q_{j^{\prime}-1}} M_{j^{\prime}-1} \times T_{q_{j^{\prime}}} M_{j^{\prime}} \cong V_{1}^{\prime} \times V_{2}^{\prime}$. Then $\mathcal{S}_{j j^{\prime}}$ is closed because it is the set of points $q \in L_{j(j+1)} \times L_{\left(j^{\prime}-1\right) j^{\prime}}$ for which $\Lambda_{2}(\underline{q})$ and $\Lambda_{1}^{\prime}(\underline{q})$ are Lagrangian, in other words such that the continuous map $\underline{q} \rightarrow$ $\Lambda_{2}(\underline{q}) \times \Lambda_{1}^{\prime}(\underline{q})$ takes values in the closed set $\mathcal{S} \times \mathcal{S}^{\prime}$. Here $\mathcal{S}, \mathcal{S}^{\prime}$ are closed by (c) above. Moreover, by the above linear algebra (b), the Lagrangian property of the intersections is equivalent to the split form of $T_{\left(q_{j}, q_{j+1}\right)} L_{j(j+1)}$ and $T_{\left(q_{j^{\prime}-1}^{\prime}, q_{j^{\prime}}\right)} L_{\left(j^{\prime}-1\right) j^{\prime}}$. This proves (i). 
Next, (ii) is similar linear algebra. Any point

$$
\left(q_{j}, q_{j+1}, q_{j^{\prime}-1}^{\prime}, q_{j^{\prime}}\right)=\left(q_{j}, \mathcal{P}_{j j^{\prime}}\left(q_{j}, q_{j^{\prime}}\right), q_{j^{\prime}}\right)
$$

has a unique lift

$$
\left(q_{j}, q_{j+1}, q_{j+1}^{\prime}, \ldots q_{j^{\prime}-1}, q_{j^{\prime}-1}^{\prime}, q_{j^{\prime}}\right) \in\left(L_{j(j+1)} \times_{H_{j+1}} \cdots \times_{H_{j^{\prime}-1}} L_{\left(j^{\prime}-1\right) j^{\prime}}\right) \cap \tilde{\mathcal{U}}_{\underline{x}, j, j^{\prime}} .
$$

Denote the symplectic spaces by $V_{i}:=T_{q_{i}} M_{i}, V_{i}^{\prime}:=T_{q_{i}^{\prime}} M_{i}$, the linear Lagrangian correspondences by $\Lambda_{i(i+1)}:=T_{\left(q_{i}, q_{i+1}\right)} L_{i(i+1)}$, and the graphs of the linearized Hamiltonian symplectomorphisms by $\operatorname{gr}_{i}:=\operatorname{graph}\left(d \phi_{\delta_{i}}^{H_{i}}\left(q_{i}\right)\right) \subset V_{i}^{-} \times V_{i}^{\prime}$. Then our choice of $\underline{H}$ guarantees that the intersection

$$
\tilde{\Lambda}_{j j^{\prime}}:=\left(\Lambda_{j(j+1)} \times \cdots \times \Lambda_{\left(j^{\prime}-1\right) j^{\prime}}\right) \pitchfork\left(V_{j} \times \mathrm{gr}_{j+1} \times \cdots \times \mathrm{gr}_{j^{\prime}-1} \times V_{j^{\prime}}\right)
$$

is transverse and the projection

$$
\Pi_{V_{j} \times V_{j^{\prime}}}: \tilde{\Lambda}_{j j^{\prime}} \stackrel{\sim}{\longrightarrow} \Lambda_{j j^{\prime}} \subset V_{j} \times V_{j^{\prime}}
$$

is an isomorphism to the Lagrangian subspace $\Lambda_{j j^{\prime}}:=T_{\left(q_{j}, q_{j^{\prime}}\right)} L_{j j^{\prime}}, \underline{\underline{x}}$. Now

$$
P:=D_{\left(q_{j}, q_{j^{\prime}}\right)} \mathcal{P}_{j j^{\prime}}: \Lambda_{j j^{\prime}} \rightarrow V_{j+1} \times V_{j^{\prime}-1}^{\prime}
$$

is the composition of the lift $\Lambda_{j j^{\prime}} \rightarrow \widetilde{\Lambda}_{j j^{\prime}}$ and the projection to $V_{j+1} \times V_{j^{\prime}-1}^{\prime}$. Hence $P \equiv 0$ is equivalent to $\Pi_{V_{j+1} \times V_{j^{\prime}-1}^{\prime}} \mid \tilde{\Lambda}_{j j^{\prime}} \equiv 0$ and hence to

$$
\tilde{\Lambda}_{j j^{\prime}} \subset V_{j} \times\{0\} \times V_{j+1}^{\prime} \times V_{j+2} \times \cdots \times V_{j^{\prime}-2}^{\prime} \times V_{j^{\prime}-1} \times\{0\} \times V_{j^{\prime}} .
$$

Since $\Lambda_{j j^{\prime}}=\Pi_{V_{j} \times V_{j^{\prime}}} \widetilde{\Lambda}_{j j^{\prime}}$ is Lagrangian, the latter is equivalent to $\Lambda_{j(j+1)} \cap\left(V_{j} \times\{0\}\right)$ and $\Lambda_{\left(j^{\prime}-1\right) j^{\prime}} \cap\left(\{0\} \times V_{j^{\prime}}\right)$ projecting to Lagrangians in $V_{j}$ and $V_{j^{\prime}}$. By (b) above, this is equivalent to $\Lambda_{j(j+1)}$ and $\Lambda_{\left(j^{\prime}-1\right) j^{\prime}}$ being of split form, and to $\Lambda_{j(j+1)} \cap\left(\{0\} \times V_{j+1}\right)$ and $\Lambda_{\left(j^{\prime}-1\right) j^{\prime}} \cap\left(V_{j^{\prime}-1}^{\prime} \times\{0\}\right)$ projecting to Lagrangians in $V_{j+1}$ and $V_{j^{\prime}-1}^{\prime}$, which is the definition of $\left(q_{j}, \mathcal{P}_{j j^{\prime}}\left(q_{j}, q_{j^{\prime}}\right), q_{j^{\prime}}\right) \in \mathcal{S}_{j j^{\prime}}$.

In the next section we will "generically" exclude quilted Floer trajectories with constant components of the following two types: Firstly, those along whose seam values we have $D \mathcal{P}_{j j^{\prime}} \neq 0$ somewhere (ie whose seam values are not entirely contained in the splitting locus); secondly, those along whose seam values $D \mathcal{P}_{j j^{\prime}} \equiv 0$ (ie with seam values entirely contained in the splitting locus) but $\Lambda_{(j+1)\left(j^{\prime}-1\right)}$ varies. This will only leave quilted Floer trajectories with constant components, for which transversality follows from transversality for the moduli space of the locally composed cyclic Lagrangian correspondence. The second part of this argument requires the following understanding of the structure of the splitting locus $\mathcal{S}_{j j^{\prime}}$, the variation of the intersection $\Lambda_{(j+1)\left(j^{\prime}-1\right)}$, and the intersection of $\mathcal{S}_{j j^{\prime}}$ with lifts of the local compositions $L_{j j^{\prime}}^{\underline{H}}$. We will describe a set of functions that cuts out the splitting locus and show that solutions with seam 
values contained in the maximally singular part of the splitting locus have constant Lagrangian intersection. In order to generically exclude other solutions, we then find Hamiltonian perturbations which ensure that the defining functions for the splitting locus pull back to regular functions (ie transverse to 0 ) on the subsets of the local compositions $L \frac{H}{j j^{\prime}}, \underline{x}$ where $\Lambda_{(j+1)\left(j^{\prime}-1\right)}$ may vary. (Note that the pullback of a regular function by an embedding fails to be regular if the image of the embedding intersects the zero set of the function nontransversely.)

Theorem 2.9 The following intersection properties hold for any pair of indices $j \triangleleft j^{\prime}$.

(a) For any $\underline{q} \in \mathcal{S}_{j j^{\prime}}$ there is an open neighborhood $\mathcal{V}_{q} \subset L_{j(j+1)} \times L_{\left(j^{\prime}-1\right) j^{\prime}}$ and smooth functions

$$
G_{n}: \mathcal{V}_{\underline{q}} \rightarrow \mathbb{R} \text { for } n=1, \ldots, n_{\max }
$$

with $n_{\max }:=\left(\operatorname{dim} M_{j}+\operatorname{dim} M_{j^{\prime}}\right)\left(\operatorname{dim} M_{j+1}+\operatorname{dim} M_{j^{\prime}-1}\right) / 4$ that cut out the splitting locus ${ }^{2}$,

$$
\mathcal{S}_{j j^{\prime}} \cap \mathcal{V}_{\underline{q}}=\bigcap_{n=1}^{n_{\max }} G_{n}^{-1}(0) .
$$

Moreover, if $\gamma:(-\epsilon, \epsilon) \rightarrow\left(M_{j} \times\left\{\tilde{q}_{j+1}\right\} \times\left\{\tilde{q}_{j^{\prime}-1}\right\} \times M_{j^{\prime}}\right) \cap \mathcal{V}_{q}$ is a smooth path contained in $\mathcal{S}_{j j^{\prime}}$ (that is, $G_{n}(\gamma(t))=0$ for all $t \in(-\epsilon, \epsilon)$ and $n=$ $1, \ldots, n_{\max }$ ), along which no $G_{n}$ has a transverse zero (ie $d G_{n}(\gamma(t)) \equiv 0$ for all $t$ and $n)$, then $\Lambda_{(j+1)\left(j^{\prime}-1\right)}(\gamma(t))$ is constant in $t \in(-\epsilon, \epsilon)$ as a subspace of $T_{\widetilde{q}_{j+1}} M_{j+1} \times T_{\widetilde{q}_{j^{\prime}-1}} M_{j^{\prime}-1}$.

(b) Fix a finite open cover

$$
\mathcal{S}_{j j^{\prime}} \subset \bigcup_{\underline{q} \in S_{j j^{\prime}}} \mathcal{V}_{\underline{q}}
$$

by subsets as in (a) with $S_{j j^{\prime}} \subset \mathcal{S}_{j j^{\prime}}$ finite, and for each $q \in S_{j j^{\prime}}$ fix functions $\left(G_{n}^{q}\right)_{n=1, \ldots, n_{\max }}$ as in (a). Then there is a dense open subset $\mathcal{H}_{j j^{\prime}}(\underline{L}) \subset \operatorname{Ham}^{*}(\underline{L})$ such that the following holds: For every $\underline{H} \in \mathcal{H}_{j j^{\prime}}(\underline{L}), \underline{x} \in \mathcal{I}(\underline{L}, \underline{H}), \underline{q} \in S_{j j^{\prime}}$, and $1 \leq n \leq n_{\max }$ the function

$$
G_{\underline{q}, \underline{\underline{H}}, \underline{x}}: \mathcal{V}_{\underline{\underline{q}, n}, \underline{H}}^{\underline{\underline{x}}} \rightarrow \mathbb{R}, \quad\left(z_{j}, z_{j^{\prime}}\right) \mapsto G \underline{\underline{q}}\left(z_{j}, \mathcal{P}_{j j^{\prime}}\left(z_{j}, z_{j^{\prime}}\right), z_{j^{\prime}}\right)
$$

${ }^{2}$ Since $L_{j(j+1)} \times L_{\left(j^{\prime}-1\right) j^{\prime}}$ splits in some other way, we could choose these functions such that $\frac{1}{4}\left(m_{j} m_{j^{\prime}-1}+m_{j^{\prime}} m_{j+1}\right)$ of them vanish identically, leaving $\frac{1}{4}\left(m_{j} m_{j^{\prime}}+m_{j+1} m_{j^{\prime}-1}\right)$ possibly nonvanishing functions. 
is transverse to 0 . It is the pullback of $G^{q}$ to the open subset of the locally composed Lagrangian $L_{j j^{\prime}}, \underline{x}$,

$\mathcal{V}_{\underline{\underline{q}}, \underline{\underline{H}}, \underline{x}}:=\left\{\left(z_{j}, z_{j^{\prime}}\right) \in L_{j j^{\prime}}^{\underline{H}, \underline{x}} \mid\left(z_{j}, \mathcal{P}_{j j^{\prime}}\left(z_{j}, z_{j^{\prime}}\right), z_{j^{\prime}}\right) \in \mathcal{V}_{\underline{q}}, d G_{n}^{\underline{q}}\left(z_{j}, \mathcal{P}_{j j^{\prime}}\left(z_{j}, z_{j^{\prime}}\right), z_{j^{\prime}}\right) \not \equiv 0\right\}$,

given by the fixed neighborhood of $\underline{q}$ and the regular locus of $G_{n}^{q}$ with respect to its full domain.

Proof Let $M, N$ be symplectic manifolds, $L \subset M \times N$ a Lagrangian submanifold, and $(m, n) \in L$ a point at which the tangent space $T_{(m, n)} L=K \times \Lambda$ splits into Lagrangian subspaces $K \subset T_{m} M$ and $\Lambda \subset T_{n} N$. Then we may symplectomorphically identify neighbourhoods $\mathcal{W}_{M} \subset M$ of $m$ and $\mathcal{W}_{N} \subset N$ of $n$ with unit balls in $B_{1}^{K} \subset K \times K^{*}$ and $B_{1}^{\Lambda} \subset \Lambda \times \Lambda^{*}$. Choosing these Darboux neighbourhoods sufficiently small ensures that $\mathcal{V}:=L \cap\left(\mathcal{W}_{M} \times \mathcal{W}_{N}\right) \cong \operatorname{graph} d F \subset B_{1}^{K} \times B_{1}^{\Lambda} \times K^{*} \times \Lambda^{*}$ is identified with the graph of the differential of some function $F: B_{1}^{K} \times B_{1}^{\Lambda} \rightarrow \mathbb{R}$. Denote by $\pi: \mathcal{V} \rightarrow B_{1}^{K} \times B_{1}^{\Lambda}$ the projection given by $\pi(p)=z$ where $p \in \mathcal{V}$ is identified with $(z, d F(z)) \in$ graph $d F$. With that notation, the tangent spaces $T_{p} L=$ graph $D^{2} F(\pi(p))$ for $p \in \mathcal{V}$ are then the graphs of the Hessian. Hence the tangent space $T_{p} L$ is of split form if and only if the Hessian $D^{2} F(\pi(p)): K \times \Lambda \rightarrow K^{*} \times \Lambda^{*}$ is of split form $D^{2} F(\pi(p))=D^{2}\left(\left.F\right|_{K}\right) \times D^{2}\left(\left.F\right|_{\Lambda}\right)$.

We will moreover identify $K \cong K^{*} \cong \mathbb{R}^{\operatorname{dim} M / 2}$ and $\Lambda \cong \Lambda^{*} \cong \mathbb{R}^{\operatorname{dim} N / 2}$ and use coordinates $(\underline{x}, y) \in K \times \Lambda$. Then $D^{2} F(\pi(p)): \mathbb{R}^{\operatorname{dim} M / 2} \times \mathbb{R}^{\operatorname{dim} N / 2} \rightarrow \mathbb{R}^{\operatorname{dim} M / 2} \times \mathbb{R}^{\operatorname{dim} N / 2}$ is a $n_{\max }:=\frac{1}{2}(\operatorname{dim} M+\operatorname{dim} N)$ square matrix, whose off-diagonal blocks give rise to the tuple of functions

$$
\left(G_{n}\right)_{n=1, \ldots, n_{\max }}: \mathcal{V} \rightarrow \mathbb{R}^{n_{\max }}, \quad p \mapsto\left(\frac{\partial^{2} F}{\partial x_{k} \partial y_{\ell}}(\pi(p))\right)_{k=1, \ldots, \frac{1}{2} \operatorname{dim} M, \ell=1, \ldots,(1 / 2) \operatorname{dim} N},
$$

which locally cut out the splitting locus,

$$
\mathcal{S} \cap \mathcal{V}=\bigcup_{n=1}^{n_{\max }} G_{n}^{-1}(0)
$$

We now apply this construction to the Lagrangian embedding

$$
L=L_{j(j+1)} \times L_{\left(j^{\prime}-1\right) j^{\prime}} \hookrightarrow M \times N
$$

given by exchange of factors into the product of $M=M_{j} \times M_{j^{\prime}}$ and $N=M_{j+1} \times M_{j^{\prime}-1}$. This yields the desired local description of the splitting locus $\mathcal{S}_{j j^{\prime}}$, consisting of points 
$\underline{q}=\left(q_{j}, q_{j+1}, q_{j^{\prime}-1}, q_{j^{\prime}}\right) \cong(m, n)$ at which both tangent spaces

$$
\begin{aligned}
T_{\left(q_{j}, q_{j+1}\right)} L_{j(j+1)} & =\Lambda_{j}^{0} \times \Lambda_{j+1}^{0} \subset T_{q_{j}} M_{j} \times T_{q_{j+1}} M_{j+1}, \\
T_{\left(q_{j^{\prime}-1}^{\prime}, q_{j^{\prime}}\right)} L_{\left(j^{\prime}-1\right) j^{\prime}} & =\Lambda_{j^{\prime}-1}^{0} \times \Lambda_{j^{\prime}}^{0} \subset T_{q_{j^{\prime}-1}} M_{j^{\prime}-1} \times T_{q_{j^{\prime}}} M_{j^{\prime}}
\end{aligned}
$$

are products of Lagrangian subspaces. In that case we have

$$
\Lambda_{(j+1)\left(j^{\prime}-1\right)}(\underline{q})=\{0\} \times \Lambda_{j+1}^{0} \times \Lambda_{j^{\prime}-1}^{0} \times\{0\} \cong T_{(m, n)} L \cap\left(\{0\} \times T_{n} N\right) .
$$

For the second part of (a) we consider a path $\gamma:(-\epsilon, \epsilon) \rightarrow\left(M \times\left\{n_{0}\right\}\right) \cap \mathcal{V}$. In the Darboux charts such a path is given by a point $y_{0} \in \Lambda$ and a smooth path $\underline{x}:(-\epsilon, \epsilon) \rightarrow K$ as $\gamma(t)=\left(\underline{x}(t), \underline{y}_{0}, \nabla F\left(\underline{x}(t), \underline{y}_{0}\right)\right)$, where $\bar{\nabla}_{\Lambda} F\left(\underline{x}(t), \underline{y}_{0}\right)$ is constant. We moreover assume $G_{n}(\gamma(t))=0$ for all $t$ and $n$, which by Proposition 2.8(a) this is equivalent to the intersection $\Lambda_{(j+1)\left(j^{\prime}-1\right)}(\gamma(t))=T_{\gamma(t)} L \cap\left(\{0\} \times T_{n_{0}} N\right)$ inducing a Lagrangian subspace in $T_{n_{0}} N$. Indeed, it is spanned by the vectors

$$
\left(0, \underline{b}, 0,\left(\sum_{i=1}^{(1 / 2) \operatorname{dim} N} b_{i} \frac{\partial^{2} F}{\partial y_{i} \partial y_{\ell}}\left(\underline{x}(t), \underline{y}_{0}\right)\right)_{\ell=1, \ldots,(1 / 2) \operatorname{dim} N}\right)
$$

for $\underline{b} \in \Lambda \cong \mathbb{R}^{(1 / 2) \operatorname{dim} N}$. Finally, we assume $d G_{n}(\gamma(t)) \equiv 0$ for all $t$ and $n$, in particular

$$
\partial_{y_{\ell}} G_{n \simeq(\kappa, i)}\left(\underline{x}(t), \underline{y}_{0}\right)=\frac{\partial^{3} F}{\partial y_{\ell} \partial y_{i} \partial x_{\kappa}}\left(\underline{x}(t), \underline{y}_{0}\right) \equiv 0
$$

for all $\ell, i, \kappa$. This guarantees that $\left(\partial^{2} F / \partial y_{i} \partial y_{\ell}\right)\left(\underline{x}(t), \underline{y}_{0}\right)$ and hence the vectors spanning $\Lambda_{(j+1)\left(j^{\prime}-1\right)}(\gamma(t))$ are independent of $t$.

Approaching (b), note that we may reformulate the claim as transversal intersection of $L_{j(j+1)} \times_{H_{j+1}} \cdots \times \times_{H_{j^{\prime}-1}} L_{\left(j^{\prime}-1\right) j^{\prime}}$ with the zero set of

$$
\widetilde{G}_{n}^{q}(\underline{z}):=G_{n}^{q}\left(z_{j}, z_{j+1}, z_{j^{\prime}-1}^{\prime}, z_{j^{\prime}}\right)
$$

on the open set

$$
\left\{\begin{array}{r|c}
\left(z_{j}, z_{j+1}, z_{j+1}^{\prime}, \ldots, z_{j^{\prime}-1}^{\prime}, z_{j^{\prime}}\right) & \left(z_{j}, z_{j+1}, z_{j^{\prime}-1}^{\prime}, z_{j^{\prime}}\right) \in \mathcal{V}_{\underline{q}}, \\
\in L_{j(j+1)} \times \ldots \times L_{\left(j^{\prime}-1\right) j^{\prime}} & d G_{n}\left(z_{j}, z_{j+1}, z_{j^{\prime}-1}^{\prime}, z_{j^{\prime}}\right) \not \equiv 0
\end{array}\right\} .
$$

The universal moduli space of regularity $m \in \mathbb{N}$ for this problem is the preimage of $\{0\} \times \Delta_{M_{j+1}} \times \cdots \Delta_{M_{j^{\prime}-1}}$ of the map

$$
\begin{aligned}
L_{j(j+1)} \times L_{(j+1)(j+2)} \cdots \times L_{\left(j^{\prime}-1\right) j^{\prime}} \times \bigoplus_{i=j+1}^{j^{\prime}-1} \mathcal{C}^{m+1}\left(\left[0, \delta_{i}\right] \times M_{i}\right) & \\
& \longrightarrow \mathbb{R} \times \prod_{i=j+1}^{j^{\prime}-1} M_{i} \times M_{i}
\end{aligned}
$$


given by $\left(z_{j}, \ldots, z_{j^{\prime}}, H_{j+1}, \ldots, H_{j^{\prime}-1}\right)$

$$
\longmapsto\left(G_{n}^{q}\left(z_{j}, z_{j+1}, z_{j^{\prime}-1}^{\prime}, z_{j^{\prime}}\right),\left(\phi_{\delta_{i}}^{H_{i}}\left(z_{i}\right), z_{i}^{\prime}\right)_{i=j+1, \ldots, j^{\prime}-1}\right) .
$$

Hence the universal moduli space is a $\mathcal{C}^{m}$ manifold if at every solution the operator

$$
\begin{aligned}
\left(v_{j}, \ldots v_{j^{\prime}}, h_{j+1}, \ldots h_{j^{\prime}-1}\right) & \\
& \longmapsto\left(d G \frac{q}{n}\left(v_{j}, v_{j+1}, v_{j^{\prime}-1}^{\prime}, v_{j^{\prime}}\right),\left(v_{i}^{\prime}-D \phi_{\delta_{i}}^{H_{i}}\left(h_{i}, v_{i}\right)\right)_{i=j+1, \ldots, j^{\prime}-1}\right)
\end{aligned}
$$

is onto. Here surjectivity in the first component is guaranteed by the condition $d \widetilde{G}_{n}^{q}(\underline{z}) \not \equiv 0$, and in the second component already $h_{i} \mapsto D \phi_{\delta_{i}}^{H_{i}}\left(h_{i}, 0\right)$ is surjective as in Theorem 2.3. Now as before the implicit function theorem and Sard-Smale theorem, using $m>\operatorname{dim} M_{j}+\operatorname{dim} M_{j^{\prime}}-1$ to satisfy the index condition, provide a dense subset of $\bigoplus_{i=j+1}^{j^{\prime}-1} \mathcal{C}^{m+1}\left(\left[0, \delta_{i}\right] \times M_{i}\right)$ for which

$$
\widetilde{G}_{n}^{\underline{q}}:\left(L_{j(j+1)} \times_{H_{j+1}} \cdots \times_{H_{j^{\prime}-1}} L_{\left(j^{\prime}-1\right) j^{\prime}}\right) \cap\left\{d \widetilde{G}_{n}^{q} \neq 0\right\} \rightarrow \mathbb{R}
$$

is transverse to 0 . Since this contains the lift of $G_{q} \underline{\underline{H}, \underline{x}}: \mathcal{V}_{\bar{q}, \underline{H}}, \underline{x} \rightarrow \mathbb{R}$, we find a dense open set of regular Hamiltonians of class $\mathcal{C}^{m+1}$ for any given $\underline{q} \in S_{j j^{\prime}}, 1 \leq n \leq N$, $\underline{x} \in \mathcal{I}(\underline{L}, \underline{H})$, and sufficiently large $m \in \mathbb{N}$. Finally, $\mathcal{C}^{1}$-small changes in $\underline{H}$ lead to small changes in intersection points and the linearized operators, hence we obtain open dense sets of regular values, and may take countable intersections to find a dense open subset $\mathcal{H}_{j j^{\prime}}(\underline{L}) \subset \operatorname{Ham}^{*}(\underline{L})$ of regular smooth Hamiltonians.

\section{Quilted Floer trajectories with constant components}

Given a cyclic generalized Lagrangian correspondence $\underline{L}$, widths $\underline{\delta}$, a regular tuple of Hamiltonian functions $\underline{H} \in \operatorname{Ham}^{*}(\underline{L})$, we now consider the Floer trajectories for some choice of almost complex structures $\underline{J}=\left(J_{j}\right)_{j=0, \ldots, r} \in \mathcal{J}_{t}(\underline{\delta})$. For any pair $\underline{x}^{-}, \underline{x}^{+} \in \mathcal{I}(\underline{L}, \underline{H})$ of generators and index $k \in \mathbb{Z}$, the moduli space of quilted Floer trajectories

$$
\begin{aligned}
\mathcal{M}^{k}\left(\underline{x}^{-}, \underline{x}^{+} ; \underline{L}, \underline{J}\right) \\
\quad:=\left\{\underline{u}=\left(u_{j}: \mathbb{R} \times\left[0, \delta_{j}\right] \rightarrow M_{j}\right)_{j=0, \ldots, r} \mid(3),(4),(5), \text { Ind } D_{\underline{u}} \bar{\partial}_{\underline{J}}=k\right\} / \mathbb{R}
\end{aligned}
$$

is the space modulo simultaneous $\mathbb{R}$-shift of tuples of perturbed holomorphic strips

$$
\bar{\partial}_{J_{j}, H_{j}} u_{j}=\partial_{s} u_{j}+J_{j}\left(\partial_{t} u_{j}-X_{H_{j}}\left(u_{j}\right)\right)=0 \quad \forall j=0, \ldots, r
$$


satisfying the seam conditions

$$
\left(u_{j}\left(s, \delta_{j}\right), u_{j+1}(s, 0)\right) \in L_{j(j+1)} \quad \forall j=0, \ldots, r, s \in \mathbb{R}
$$

as well as uniform limits

$$
\lim _{s \rightarrow \pm \infty} u_{j}(s, \cdot)=x_{j}^{ \pm} \quad \forall j=0, \ldots, r .
$$

Moreover, we fixed the index of the linearized operator - as explained in the following. By standard local action arguments as in Floer [2] any such solution also has finite energy, and exponential decay analysis as in [11] shows that any solution is of Sobolev regularity $W^{1, p}$ relative to the limits for any $p>2$ in the following sense: If we trivially extend $x_{j}^{ \pm}$to maps $\mathbb{R} \times\left[0, \delta_{j}\right] \rightarrow M_{j}$, then there exists $R>0$ such that $u_{j}( \pm s, t)$ takes values in an exponential ball (with respect to some and hence any metric on $\left.M_{j}\right)$ around $x_{j}^{ \pm}(s, t)$ for $\pm s>R$, and such that for each $j=0, \ldots, r$ we have

$$
\left((s, t) \mapsto \exp _{x_{j}^{ \pm}(t)}^{-1}\left(u_{j}( \pm s, t)\right)\right) \in W^{1, p}\left([R, \infty) \times\left[0, \delta_{j}\right], x_{j}^{ \pm *} T M_{j}\right) .
$$

With this, the moduli space of Floer trajectories can be identified with the $\mathbb{R}$-quotient of the zero set of a section $\bar{\partial}_{\underline{J}}: \mathcal{B} \rightarrow \mathcal{E}$ of a Banach bundle, where

$$
\mathcal{B}:=\left\{\underline{u}=\left(u_{j} \in W_{\mathrm{loc}}^{1, p}\left(\mathbb{R} \times\left[0, \delta_{j}\right], M_{j}\right)\right)_{j=0, \ldots, r} \mid(4),(6)\right\},
$$

$\mathcal{E} \rightarrow \mathcal{B}$ is the Banach bundle with fibers $\mathcal{E}_{\underline{u}}=\bigoplus_{j=0}^{r} L^{p}\left(\mathbb{R} \times\left[0, \delta_{j}\right], u_{j}^{*} T M_{j}\right)$, and $\bar{\partial}_{\underline{J}}: \mathcal{B} \rightarrow \mathcal{E}$ is the $\left(\mathbb{R}\right.$-invariant) Cauchy-Riemann operator $\bar{\partial}_{\underline{J}}(\underline{u})=\left(\bar{\partial}_{J_{j}}, H_{j} u_{j}\right)_{j=0, \ldots, r}$. In [10] we proved that $\bar{\partial}_{\underline{J}}$ is a Fredholm section, and in the definition of the moduli space $\mathcal{M}^{k}\left(\underline{x}^{-}, \underline{x}^{+}\right)$we fix the Fredholm index of its linearization $D_{\underline{u}} \bar{\partial}_{\underline{J}}: T_{\underline{u}} \mathcal{B} \rightarrow \mathcal{E}_{\underline{u}}$. In order to achieve transversality of the section $s$, we now restrict ourselves to a further dense open subset of Hamiltonian perturbations, as constructed in Section 2.

Definition 3.1 Given a cyclic generalized Lagrangian correspondence $\underline{L}$ and widths $\underline{\delta}$, let

$$
\mathcal{H}_{\text {reg }}(\underline{L})=\bigcap_{j, j^{\prime}} \mathcal{H}_{j j^{\prime}}(\underline{L}) \subset \mathcal{H}_{t}(\underline{\delta})
$$

be the intersection over all pairs of indices $j \triangleleft j^{\prime}$ of the dense open subsets of regular Hamiltonians for some choices of covers of $\mathcal{S}_{j j^{\prime}}$ as in Theorem 2.9.

We now prove the main result. 
Theorem 3.2 For any cyclic generalized Lagrangian correspondence $\underline{L}$ and any choice of widths $\underline{\delta}$ and regular Hamiltonians $\underline{H} \subset \mathcal{H}_{\text {reg }}(\underline{L})$, there exists a comeagre ${ }^{3}$ subset $\mathcal{J}_{\text {reg }}(\underline{L} ; \underline{H}) \subset \mathcal{J}_{t}(\underline{\delta})$ such that for all $\underline{J} \in \mathcal{J}_{\text {reg }}(\underline{L} ; \underline{H}), \underline{x}^{ \pm} \in \mathcal{I}(\underline{L}, \underline{H})$, and $k \in \mathbb{Z}$ the Cauchy-Riemann section $\bar{\partial}_{\underline{J}}: \mathcal{B} \rightarrow \mathcal{E}$ defined above is transverse to the zero section.

Remark 3.3 In fact, we prove that for generic perturbation data $\underline{H} \subset \operatorname{Ham}^{*}(\underline{L})$ and $\underline{J} \in \mathcal{J}_{t}^{\text {reg }}(\underline{L} ; \underline{H})$ any solution $\underline{u} \in \mathcal{M}^{k}\left(\underline{x}^{-}, \underline{x}^{+} ; \underline{L}, \underline{J}\right)$ with some constant components has split linearized seam conditions between constant and nonconstant components in the following sense: If $\partial_{s} u_{j} \not \equiv 0$ and $u_{j+1}(s, t)=x_{j+1}(t)$ for all $(s, t) \in \mathbb{R} \times[0,1]$, then $T_{\left(u_{j}\left(s, \delta_{j}\right), x_{j+1}(0)\right)} L_{j(j+1)}=\Lambda_{j}(s) \times \Lambda_{j+1}$ splits into two families of Lagrangian subspaces

$$
\begin{aligned}
& \Lambda_{j}(s)=\Pi_{T M_{j}}\left(T_{\left(u_{j}\left(s, \delta_{j}\right), x_{j+1}(0)\right)} L_{j(j+1)} \cap\left(T_{u_{j}\left(s, \delta_{j}\right)} M_{j} \times\{0\}\right)\right), \\
& \Lambda_{j+1}=\Pi_{T M_{j+1}}\left(T_{\left(u_{j}\left(s, \delta_{j}\right), x_{j+1}(0)\right)} L_{j(j+1)} \cap\left(\{0\} \times T_{x_{j+1}(0)} M_{j+1}\right)\right),
\end{aligned}
$$

of which the second is constant. The analogous statement holds for $\partial_{s} u_{j} \equiv 0$ and $\partial_{s} u_{j+1} \not \equiv 0$.

Proof Since $\mathcal{I}(\underline{L}, \underline{H})$ has finitely many elements (due to the transversality in Theorem 2.3 and compactness of the Lagrangian correspondences), and countable intersections of comeagre sets are comeagre in the complete metric space $\mathcal{J}_{t}(\underline{\delta})$, it suffices to consider a single pair $\underline{x}_{ \pm} \in \mathcal{I}(\underline{L}, \underline{H})$ and indices $k \leq k_{0}$ for some fixed $k_{0} \in \mathbb{N}$.

The standard universal moduli space approach, using unique continuation for each strip separately, as discussed in the proof of [10, Theorem 5.2.4.], provides a comeagre subset in $\mathcal{J}_{t}(\underline{L})$ for which the section $s$ is transverse at all zeros $\underline{u}$ for which $\partial_{s} u_{i} \not \equiv 0$ for all $i=0, \ldots, r$. In addition, $s$ is automatically transverse at any completely constant solution $\underline{u} \equiv \underline{x}^{+}=\underline{x}^{-}$, by a general argument (using spectral analysis, elliptic regularity, and Sobolev embeddings) as in eg Salamon [8, Lemma 2.4] and Donaldson [1, Chapter 3].

More precisely, the linearized operator at such a solution $\underline{u} \equiv\left(x_{j}\right) \in \mathcal{I}(\underline{L}, \underline{H})$ has the form $\partial_{s}+A: T_{\underline{u}} \mathcal{B} \rightarrow \mathcal{E}_{\underline{u}}$, where $T_{\underline{u}} \mathcal{B} \subset L^{p}\left(\mathbb{R}, W^{p}\right)$ is a subset of paths in

$$
\begin{aligned}
W^{p}:=\left\{\left(\xi_{j} \in W^{1, p}\left(\left[0, \delta_{j}\right], x_{j}^{*} T M_{j}\right)\right)_{j=0, \ldots, r} \mid\right. \\
\left.\left(\xi_{j}\left(\delta_{j}\right), \xi_{j+1}(0)\right) \in T_{\left(x_{j}\left(\delta_{j}\right), x_{j+1}(0)\right)} L_{j(j+1)} \forall j\right\}
\end{aligned}
$$

${ }^{3}$ A subset of a topological space is comeagre (or residual) if it is the intersection of countably many open dense subsets. Many authors in symplectic topology would use the term "Baire second category", which however in classical Baire theory (see Royden [7, Chapter 7.8]) denotes more generally subsets that are not meagre, ie not the complement of a comeagre subset. Baire's Theorem applies to complete metric spaces such as the spaces of smooth almost complex structures considered here, and implies that every comeagre set is dense. 
and $A=\left(J_{j}\left(x_{j}^{ \pm}\right) \partial_{t}\right)_{j=0, \ldots, r}$ is independent of $s \in \mathbb{R}$. This operator extends to an invertible self-adjoint operator $\tilde{A}: W^{2} \rightarrow H$, where $H$ is the $L^{2}$-closure of $W^{p}$. Now $\partial_{s}+\widetilde{A}: L^{2}\left(\mathbb{R}, W^{2}\right) \cap W^{1,2}(\mathbb{R}, H) \rightarrow L^{2}(\mathbb{R}, H)$ is an isomorphism by Robbin and Salamon [6, Proposition 3.1.12]. Here the domain and target are the $W^{1,2}$, resp. $L^{2}$, closures of the compactly supported smooth maps in $T_{\underline{u}} \mathcal{B}$ resp. $\mathcal{E}_{\underline{u}}$. Now a standard method (worked out eg in $[8 ; 1]$ ) allows to transfer the isomorphism result to the case of $W^{1, p}$ domain and $L^{p}$ target. In our case, we can copy the proof of [8, Lemma 2.4] word-for-word and simply replace the elliptic regularity and estimates for the Cauchy-Riemann operator $\partial_{s}+A$ on finite cylinders with those for the tuple of Cauchy-Riemann operators $\partial_{s}+A$ on tuples of finite strips with linearized Lagrangian seam conditions.

It remains to consider solutions $\underline{u}$ for which a proper subset $u_{i}$ for $i \in I \subset\{0, \ldots, r\}$ of components is constant. Here and in the following we call a component $u_{i}$ constant if $\partial_{s} u_{i}=0$, and hence $\partial_{t} u_{i}=X_{H_{i}}\left(u_{i}\right)$, so $u_{i}$ is a Hamiltonian trajectory in $t$, independent of $s$. A necessary condition for such solutions to exist is $x_{i}^{-}=x_{i}^{+}$for all $i \in I$, and hence the locally composed cyclic Lagrangian correspondences $\underline{L}^{I,} \underline{H}^{,} \underline{x}^{+}=\underline{L}^{I,}, \underline{H}, \underline{x}^{-}$ are the same. Note that any solution $\underline{u} \in \mathcal{M}^{k}\left(\underline{x}^{-}, \underline{x}^{+} ; \underline{L}, \underline{J}\right)$ with the $I$ components constant induces a solution $\left(u_{i}\right)_{i \in I^{C}} \in \mathcal{M}^{k}\left(\left(x_{i}^{-}\right)_{i \in I^{C}},\left(x_{i}^{+}\right)_{i \in I^{C}} ; \underline{L}^{I, \underline{H}}, \underline{x}^{ \pm},\left(J_{i}\right)_{i \in I^{C}}\right)$ in the moduli space of same index (see [10,3.1.8] for the index calculation) for the locally composed correspondence. Indeed, for consecutive pairs of indices $j \triangleleft j^{\prime} \in I^{C}$ we have

$$
\begin{aligned}
\left(u_{j}\left(s, \delta_{j}\right), x_{j+1}(0), x_{j+1}\left(\delta_{j+1}\right), \ldots,\right. & \left.x_{j^{\prime}-1}\left(\delta_{j^{\prime}-1}\right), u_{j^{\prime}}(s, 0)\right) \\
& \in\left(L_{j(j+1)} \times_{H_{j+1}} \cdots \times_{H_{j^{\prime}-1}} L_{\left(j^{\prime}-1\right) j^{\prime}}\right) \cap \tilde{\mathcal{U}}_{\underline{x}, j, j^{\prime}} .
\end{aligned}
$$

The converse is rarely true since the lifts from $L_{j j^{\prime}}^{\underline{H} \underline{x}^{ \pm}}$to $L_{j(j+1)} \times_{H_{j+1}} \cdots \times_{H_{j^{\prime}-1}}$ $L_{\left(j^{\prime}-1\right) j^{\prime}}$ may not be constant. Part of this is encoded by the lift map

$$
\mathcal{P}_{j j^{\prime}}: L_{j j^{\prime}}^{\underline{H} \underline{x}^{ \pm}} \rightarrow M_{j+1} \times M_{j^{\prime}-1}
$$

from Definition 2.7. In the following six steps we substantiate the intuition laid out in the introduction: Automatic transversality can fail for Floer trajectories with a mix of constant and nonconstant components. However, those are in fact nongeneric solutions. We denote by $\mathcal{J}_{t}^{\ell}(\underline{\delta})$ the closure of $\mathcal{J}_{t}(\underline{\delta})$ in the topology of $C^{\ell}$-maps $\left[0, \delta_{j}\right] \times T M_{j} \rightarrow T M_{j}$.

Step 0 In preparation we need to fix some choices for each pair of indices $j \triangleleft j^{\prime}$. 
Firstly, we fix a metric on each $M_{i}$. Then, since $L \frac{H}{j j}, \underline{x}^{ \pm}$is compact, we may fix an open cover

$$
\mathcal{P}_{j j^{\prime}}\left(L \underline{H}_{j j^{\prime}}, \underline{x}^{ \pm}\right) \subset \bigcup_{\underline{p} \in C_{j j^{\prime}}} \mathcal{W}_{\underline{p}}
$$

by a finite number (indexed by $C_{j j^{\prime}} \subset M_{j+1} \times M_{j^{\prime}-1}$ ) of exponential balls $\mathcal{W}_{\underline{p}} \subset$ $M_{j+1} \times M_{j^{\prime}-1}$ on which

$$
\exp _{\underline{p}}^{-1}: \mathcal{W}_{\underline{p}} \rightarrow B_{\epsilon_{\underline{p}}}(0) \subset T_{\underline{p}}\left(M_{j+1} \times M_{j^{\prime}-1}\right)
$$

is a diffeomorphism. We also fix a collection of 1-dimensional subspaces $\left(Z_{m}\right)$ for $m=1, \ldots, \operatorname{dim} M_{j+1}+\operatorname{dim} M_{j^{\prime}-1}$ spanning $T_{\underline{p}}\left(M_{j+1} \times M_{j^{\prime}-1}\right)$ for each $\underline{p} \in C_{j j^{\prime}}$. Secondly, as in Theorem 2.9 we fix a finite open cover $\mathcal{S}_{j j^{\prime}} \subset \bigcup_{\underline{q} \in S_{j j^{\prime}}} \mathcal{V}_{\underline{q}}$ and choose $\underline{H}$ such that the submanifold

$$
\left\{\underline{z} \in \mathcal{V}_{\underline{q}} \mid G_{n}(\underline{z})=0, d G_{n}(\underline{z}) \neq 0\right\} \subset L_{j(j+1)} \times L_{\left(j^{\prime}-1\right) j^{\prime}}
$$

is transverse to $\widetilde{L}_{j j^{\prime}}^{\underline{H}, \underline{x}^{ \pm}}$for every $\underline{q} \in S_{j j^{\prime}}$ and $n=1, \ldots, N$.

Step 1.a We start by reviewing the regularity of the linearized operator at solutions without constant components, more precisely we prove the following:

For every integer $\ell>k_{0}$ there exists a comeagre subset $\mathcal{J}_{1}^{\ell} \subset \mathcal{J}_{t}^{\ell}(\underline{\delta})$ such that for any $\underline{J} \in \mathcal{J}_{1}^{\ell}$ the linearized operator $D_{\underline{u}} \bar{\partial}_{\underline{J}}$ is surjective for all $\underline{u} \in \bigcup_{k \leq k_{0}} \mathcal{M}^{k}\left(\underline{x}^{-}, \underline{x}^{+} ; \underline{L}, \underline{J}\right)$ with no constant components.

This is what the arguments of [10] actually prove. To be precise, we consider the operator $\mathcal{J}_{t}^{\ell}(\underline{\delta}) \times\left.\mathcal{B}_{\mathrm{nc}} \rightarrow \mathcal{E}\right|_{\mathcal{B}_{\mathrm{nc}}},(\underline{J}, \underline{u}) \mapsto \bar{\partial}_{\underline{J}} \underline{u}$ on the open subset

$$
\mathcal{B}_{\mathrm{nc}}:=\left\{\underline{u} \in \mathcal{B} \mid \partial_{s} u_{i} \not \equiv 0 \forall i=0, \ldots, r\right\} \subset \mathcal{B} .
$$

This operator cuts out the universal moduli space and is a $\mathcal{C}^{\ell}$ section of a Banach bundle whose linearized operator at a zero $\bar{\partial}_{\underline{J}} \underline{u}=0$ is

$$
\left(\underline{K}=\left(K_{i}\right)_{i=0, \ldots, r}, \underline{\xi}\right) \mapsto\left(D_{\underline{u}} \bar{\partial} \underline{J}\right) \underline{\xi}-\left(K_{i} J_{i} \partial_{s} u_{i}\right)_{i=0, \ldots, r} .
$$

Here the second summand is already surjective by the same arguments as in Floer, Hofer and Salamon [3]. Indeed, the unique continuation theorem applies to the interior of every single nonconstant strip $u_{i}: \mathbb{R} \times\left(0, \delta_{i}\right) \rightarrow M_{i}$ and implies that the set of regular points, $\left(s_{0}, t_{0}\right) \in \mathbb{R} \times\left(0, \delta_{i}\right)$ with $\partial_{s} u_{i}\left(s_{0}, t_{0}\right) \neq 0$ and $u_{i}^{-1}\left(u_{i}(\mathbb{R} \cup\{ \pm \infty\}), t_{0}\right)=\left\{\left(s_{0}, t_{0}\right)\right\}$, is open and dense. This suffices to prove surjectivity by contradiction. So by the implicit function theorem $\left\{(\underline{J}, \underline{u}) \mid \bar{\partial}_{\underline{J}} \underline{u}=0\right\}$ is a $\mathcal{C}^{\ell}$ Banach manifold. Its projection to $\mathcal{J}_{t}^{\ell}(\underline{\delta})$ is a Fredholm map of class $\mathcal{C}^{\ell}$ and index Ind $D_{\underline{u}} \leq k_{0} \leq \ell-1$. Hence, by the Sard-Smale theorem, the set of regular values, which coincides with the set $\underline{J} \in \mathcal{J}_{t}^{\ell}(\underline{\delta})$ such that $D_{\underline{u}} \bar{\partial}_{\underline{J}}$ is surjective for all solutions $\underline{u}$, is comeagre as claimed. 
Step 1.b Next, a similar argument provides the following regularity of the linearized operator for the locally composed cyclic generalized Lagrangian correspondences:

For every proper subset $I \subset\{0, \ldots, r\}$ such that $\left(x_{i}^{-}\right)_{i \in I}=\left(x_{i}^{+}\right)_{i \in I}$ and every integer $\ell>k_{0}$ there exists a comeagre subset $\mathcal{J}_{1, I}^{\ell} \subset \mathcal{J}_{t}^{\ell}(\underline{\delta})$ such that for any $\underline{J} \in \mathcal{J}_{1, I}^{\ell}$ and

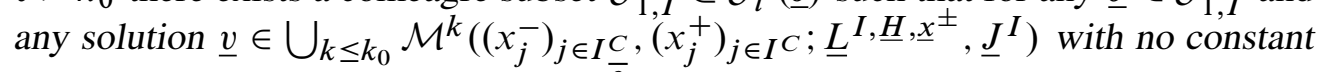
components the linearized operator $D_{\underline{v}} \bar{\partial}_{\underline{J}} I$ is surjective.

The locally composed cyclic Lagrangian correspondence $\underline{L}^{I, \underline{H}, \underline{x}^{ \pm}}$consists of smooth, yet not compact, Lagrangian submanifolds. However, the compactness is not relevant for the universal moduli space arguments. Hence as in Step 1.a we find a comeagre subset of the $\mathcal{C}^{\ell}$-closure of $\bigoplus_{j \in I^{C}} \mathcal{C}^{\infty}\left(\left[0, \delta_{j}\right], \mathcal{J}\left(M_{j}, \omega_{j}\right)\right)$ with the transversality properties. Then we let $\mathcal{J}_{1, I}^{\ell}$ be the preimage under the projection $\underline{J} \rightarrow \underline{J}^{I}=\left(J_{j}\right)_{j \in I^{C}}$.

Step 2 In this first nonstandard step we show that for quilted Floer trajectories with respect to generic almost complex structures the differential $D \mathcal{P}_{j j^{\prime}}$ of the lift map from Definition 2.7 vanishes along the seams bounding constant components. More precisely:

For every integer $\ell>k_{0}$ and pair of indices $j \triangleleft j^{\prime}$ such that $x_{i}^{-}=x_{i}^{+}$for $i=$ $j+1, \ldots, j^{\prime}-1$ there exists a comeagre subset $\mathcal{J}_{2, j, j^{\prime}} \subset \mathcal{J}_{t}^{\ell}(\underline{\delta})$ such that for any $\underline{J} \in \mathcal{J}_{2, j, j^{\prime}}^{\ell}$ and $\underline{u} \in \bigcup_{k \leq k_{0}} \mathcal{M}^{k}\left(\underline{x}^{-}, \underline{x}^{+} ; \underline{L}, \underline{J}\right)$ with $u_{j+1}, \ldots, u_{j^{\prime}-1}$ constant we have $D_{\left(u_{j}\left(s, \delta_{j}\right), u_{j^{\prime}}(s, 0)\right)} \mathcal{P}_{j j^{\prime}}=0$ for all $s \in \mathbb{R}$.

Given $\ell, j, j^{\prime}$ we set $I:=\left\{j+1, \ldots, j^{\prime}-1\right\}$ and start by proving an intermediate Lemma, which asserts emptiness of the moduli spaces of quilted Floer trajectories for $\underline{L}^{I, \underline{H}, \underline{x}^{ \pm}}$with $D \mathcal{P}_{j j^{\prime}} \not \equiv 0$ but a weak form of constant lifts $\mathcal{P}_{j j^{\prime}}$ at sufficiently many points along the seam. (Note that we make sure to only introduce a countable set of data $\left(k, \underline{p}, Z_{m},\left(s_{0}, \ldots, s_{k}\right)\right)$ since we will later need to take the intersection of comeagre subsets for each choice and wish to still obtain a comeagre set of $\underline{J}$.)

Lemma for Step 2 Fix a choice of $k \leq k_{0}$, one of the fixed centers of exponential balls $\underline{p} \in C_{j j^{\prime}}$, and one of the fixed 1-dimensional subspaces $Z_{m} \subset T_{\underline{p}}\left(M_{j+1} \times M_{j^{\prime}-1}\right)$. $\overline{\text { For all }} s \in \mathbb{R}$ and Floer trajectories

$$
\underline{v} \in \mathcal{M}^{k}\left(\left(x_{i}^{-}\right)_{i \in I^{C}},\left(x_{i}^{+}\right)_{i \in I^{C}} ; \underline{L}^{I, \underline{H}, \underline{x}^{ \pm}}, \underline{J}^{I}\right)
$$

such that $\mathcal{P}_{j j^{\prime}}\left(v_{j}\left(s, \delta_{j}\right), v_{j^{\prime}}(s, 0)\right) \in \mathcal{W}_{\underline{p}}$ denote

$$
\begin{aligned}
\mathfrak{z}(\underline{v}, s) & :=\Pi_{Z_{m}} \exp _{\underline{p}}^{-1}\left(\mathcal{P}_{j j^{\prime}}\left(v_{j}\left(s, \delta_{j}\right), v_{j^{\prime}}(s, 0)\right)\right), \\
\mathfrak{D}(\underline{v}, s) & :=\Pi_{Z_{m}} \circ D \exp _{\underline{p}}^{-1} \circ D_{\left(v_{j}\left(s, \delta_{j}\right), v_{j^{\prime}}(s, 0)\right)} \mathcal{P}_{j j^{\prime}} .
\end{aligned}
$$


Then for any tuple of rationals $s_{0}<\cdots<s_{k+1} \in \mathbb{Q}$ there exists a comeagre subset $\mathcal{J}\left(\underline{p}, m, s_{0}, \ldots, s_{k+1}\right) \subset \mathcal{J}_{t}^{\ell}(\underline{\delta})$ such that for any $\underline{J} \in \mathcal{J}\left(\underline{p}, m, s_{0}, \ldots, s_{k+1}\right)$ there exists no Floer trajectory

$$
\underline{v} \in \mathcal{M}^{k}\left(\left(x_{i}^{-}\right)_{i \in I^{C}},\left(x_{i}^{+}\right)_{i \in I^{C}} ; \underline{L}^{I, \underline{H}, \underline{x}^{ \pm}}, \underline{J}^{I}\right)
$$

satisfying $\mathcal{P}_{j j^{\prime}}\left(v_{j}\left(s_{l}, \delta_{j}\right), v_{j^{\prime}}\left(s_{l}, 0\right)\right) \in \mathcal{W}_{\underline{p}}$ and $\mathfrak{D}\left(\underline{v}, s_{l}\right) \neq 0$ for all $l=0, \ldots, k+1$, and moreover

$$
\mathfrak{z}\left(\underline{v}, s_{0}\right)=\mathfrak{z}\left(\underline{v}, s_{1}\right)=\cdots=\mathfrak{z}\left(\underline{v}, s_{l}\right) .
$$

The proof is by a universal moduli space argument. Let $\mathcal{B}$ be the Banach manifold as

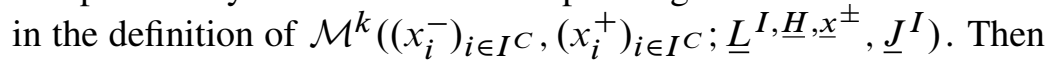

$$
\mathcal{B}^{\prime}:=\left\{\begin{array}{l|l}
\underline{v} \in \mathcal{B} & \begin{array}{l}
\mathcal{P}_{j j^{\prime}}\left(v_{j}\left(s_{l}, \delta_{j}\right), v_{j^{\prime}}\left(s_{l}, 0\right)\right) \in \mathcal{W}_{p} \\
\mathfrak{D}\left(\underline{v}, s_{l}\right) \neq 0 \quad \forall 0 \leq l \leq k+1
\end{array} \quad \forall 0 \leq l \leq k+1,
\end{array}\right\}
$$

is an open subset of $\mathcal{B}$, and

$$
s(\underline{J}, \underline{v}):=\left(\bar{\partial}_{\underline{J}^{I}}(\underline{v}),\left(\mathfrak{z}\left(\underline{v}, s_{l}\right)-\mathfrak{z}\left(\underline{v}, s_{0}\right)\right)_{l=1, \ldots, k+1}\right)
$$

defines a $\mathcal{C}^{\ell}$ section of the bundle $\left.\mathcal{E}\right|_{\mathcal{B}^{\prime}} \times\left(Z_{m}\right)^{k+1} \rightarrow \mathcal{J}_{t}^{\ell}(\underline{\delta}) \times \mathcal{B}^{\prime}$. Its linearized operator at a zero maps $\left(\underline{K}=\left(K_{i}\right)_{i=0, \ldots, r}, \underline{\xi}=\left(\xi_{i}\right)_{i \in I^{C}}\right)$ to

$$
\left(\begin{array}{c}
\left(D_{\underline{v} \bar{\partial}^{I}}\right) \underline{\xi}-\left(K_{i} J_{i} \partial_{s} v_{i}\right)_{i \in I^{C}} \\
\left(\mathfrak{D}\left(\underline{v}, s_{l}\right)\left(\xi_{j}\left(s_{l}, \delta_{j}\right), \xi_{j^{\prime}}\left(s_{l}, 0\right)\right)-\mathfrak{D}\left(\underline{v}, s_{0}\right)\left(\xi_{j}\left(s_{0}, \delta_{j}\right), \xi_{j^{\prime}}\left(s_{0}, 0\right)\right)\right)_{l=1, \ldots, k+1}
\end{array}\right) .
$$

Here the second summand in the first component is surjective by the same arguments as in Step 1.a, using just the freedom in $\underline{K}$. The second component is surjective since by definition of $\mathcal{B}^{\prime}$ each map

$$
\mathfrak{D}\left(\underline{v}, s_{l}\right): T_{\left(v_{j}\left(s_{l}, \delta_{j}\right), v_{j^{\prime}}\left(s_{l}, 0\right)\right)} L_{j j^{\prime}}^{\underline{H} \underline{x}^{ \pm}} \rightarrow Z_{m}
$$

is nonzero, ie surjective onto this one dimensional subspace, and $\underline{\xi}$ can be chosen to assume any given tuple of values on the linearized Lagrangian correspondence $T L \frac{H}{j j^{\prime}} \underline{x}^{ \pm}$ at distinct $s_{1}, \ldots, s_{k+1} \in \mathbb{R}$. So by the implicit function theorem $\{(\underline{J}, \underline{v}) \mid s(\underline{J}, \underline{v})=0\}$ is a $\mathcal{C}^{\ell}$ Banach manifold. Its projection to $\mathcal{J}_{t}^{\ell}(\underline{\delta})$ is a Fredholm map of class $\mathcal{C}^{\ell}$ and index Ind $D_{\underline{v}}-(k+1)=-1$. Hence, by the Sard-Smale theorem, the set of regular values is comeagre. Finally, since the index is negative, the set of solutions for a regular $\underline{J}$ is empty, which proves the Lemma.

We now obtain a comeagre subset $\mathcal{J}_{2, j, j^{\prime}}^{\ell} \subset \mathcal{J}_{t}^{\ell}(\underline{\delta})$ by taking the countable intersection of the comeagre sets $\mathcal{J}\left(\underline{p}, m, s_{0}, \ldots, s_{k+1}\right)$ given by the Lemma for each 
choice of $p, m$, and finite subset $\left\{s_{0}, \ldots, s_{k+1}\right\} \subset \mathbb{Q}$. Then suppose by contradiction that for some $\underline{J} \in \mathcal{J}_{2, j, j^{\prime}}^{\ell}$ we have a solution $\underline{u} \in \mathcal{M}^{k}\left(\underline{x}^{-}, \underline{x}^{+} ; \underline{L}, \underline{J}\right)$ for some $k \leq k_{0}$ with $u_{j+1}, \ldots, u_{j^{\prime}-1}$ constant but $D_{\left(u_{j}\left(s_{0}, \delta_{j}\right), u_{j^{\prime}}\left(s_{0}, 0\right)\right)} \mathcal{P}_{j j^{\prime}} \neq 0$ for some $s_{0} \in \mathbb{R}$. As discussed at the beginning of the proof of this theorem, $\underline{u}$ induces a solution $\underline{v}:=\left(u_{i}\right)_{i \in I^{C}} \in \mathcal{M}^{k}\left(\left(x_{i}^{-}\right)_{i \in I^{C}},\left(x_{i}^{+}\right)_{i \in I^{C}} ; \underline{L}^{I, \underline{H}, \underline{x}^{ \pm}}, \underline{J}^{I}\right)$ such that $\mathcal{P}_{j j^{\prime}}\left(v_{j}\left(s, \delta_{j}\right), v_{j^{\prime}}(s, 0)\right)=\left(u_{j+1}(s, 0), u_{j^{\prime}-1}\left(s, \delta_{j^{\prime}-1}\right)\right)$ is constant in $s \in \mathbb{R}$. Moreover, for some $s_{0} \in \mathbb{R}$ we have $D_{\left(v_{j}\left(s_{0}, \delta_{j}\right), v_{j^{\prime}}\left(s_{0}, 0\right)\right)} \mathcal{P}_{j j^{\prime}} \neq 0$. Since this is an open condition, we may also find $s_{0} \in \mathbb{Q}$ with the same nonvanishing. Then we have $\mathcal{P}_{j j^{\prime}}\left(v_{j}\left(s_{0}, \delta_{j}\right), v_{j^{\prime}}\left(s_{0}, 0\right)\right) \in \mathcal{W}_{\underline{p}}$ for some $\underline{p} \in C_{j j^{\prime}}$ and

$$
\mathfrak{D}\left(\underline{v}, s_{0}\right)=\Pi_{Z_{m}} \circ D \exp _{\underline{p}}^{-1} \circ D_{\left(v_{j}\left(s_{0}, \delta_{j}\right), v_{j^{\prime}}\left(s_{0}, 0\right)\right)} \mathcal{P}_{j j^{\prime}} \neq 0
$$

for one of the spanning subspaces $Z_{m} \subset T_{p}\left(M_{j+1} \times M_{j^{\prime}-1}\right)$. Again, these are open conditions, so we may find rational numbers $s_{0}<s_{1}<\cdots<s_{k+1}$ with the same properties. This contradicts the Lemma since $\mathfrak{z}(\underline{v}, s)=\mathcal{P}_{j j^{\prime}}\left(v_{j}\left(s, \delta_{j}\right), v_{j^{\prime}}(s, 0)\right)=$ $\left(x_{j+1}(0), x_{j^{\prime}-1}\left(\delta_{j^{\prime}-1}\right)\right)$ is independent of $s \in\left\{s_{0}, \ldots, s_{l}\right\}$.

Step 3 Extending Step 2, we show that for quilted Floer trajectories with respect to generic almost complex structures in fact the splitting condition of Proposition 2.8 on the linearized seam conditions holds along the seams bounding constant components. More precisely:

For every integer $\ell>k_{0}$ and pair of indices $j \triangleleft j^{\prime}$ such that $x_{i}^{-}=x_{i}^{+}=: x_{i}$ for $i=j+1, \ldots, j^{\prime}-1$ there exists a comeagre subset $\mathcal{J}_{3, j, j^{\prime}}^{\ell} \subset \mathcal{J}_{t}^{\ell}(\underline{\delta})$ such that for any $\underline{J} \in \mathcal{J}_{3, j, j^{\prime}}^{\ell}$ and $\underline{u} \in \bigcup_{k \leq k_{0}} \mathcal{M}^{k}\left(\underline{x}^{-}, \underline{x}^{+} ; \underline{L}, \underline{J}\right)$ with $u_{j+1}, \ldots, u_{j^{\prime}-1}$ constant the intersection at $y(s):=\left(u_{j}\left(s, \delta_{j}\right), x_{j+1}(0), x_{j^{\prime}-1}\left(\delta_{j^{\prime}-1}\right), u_{j^{\prime}}(s, 0)\right)$

$$
T_{y(s)}\left(L_{j(j+1)} \times L_{\left(j^{\prime}-1\right) j^{\prime}}\right) \cap\left(\{0\} \times T_{x_{j+1}(0)} M_{j+1} \times T_{x_{j^{\prime}-1}\left(\delta_{j^{\prime}-1}\right)} M_{j^{\prime}-1} \times\{0\}\right)
$$

projects to a Lagrangian subspace $T_{x_{j+1}(0)} M_{j+1} \times T_{x_{j^{\prime}-1}\left(\delta_{j^{\prime}-1}\right)} M_{j^{\prime}-1}$ that is independent of $s \in \mathbb{R}$.

Given $\ell, j, j^{\prime}$ we set $I:=\left\{0, \ldots, j, j^{\prime}, \ldots, r\right\}$ and start by proving an intermediate Lemma which asserts emptiness of the moduli spaces of quilted Floer trajectories for $\underline{L}^{I, \underline{H}, \underline{x}^{ \pm}}$with $d G_{n} \not \equiv 0$ but $G_{n}=0$ at sufficiently many points along the seam. This will be relevant since by Theorem 2.9 the splitting locus is locally given by the intersection of the zero sets $G_{n}^{-1}(0)$, and since $d G_{n} \equiv 0$ along a path in the splitting locus ensures $s$-independence of the Lagrangian subspace of $T M_{j+1} \times T M_{j^{\prime}-1}$ that arises from the splitting. 
Lemma for Step 3 For every $k \leq k_{0}, q \in S_{j j^{\prime}}, 1 \leq n \leq N$, and tuple of rationals $s_{0}<\cdots<s_{k} \in \mathbb{Q}$ there exists a comeagre subset $\mathcal{J}\left(\underline{q}, n, s_{0}, \ldots, s_{k}\right) \subset \mathcal{J}_{t}^{\ell}(\underline{\delta})$ such that for $\underline{J} \in \mathcal{J}\left(\underline{q}, n, s_{0}, \ldots, s_{k}\right)$ there exists no solution

$$
\underline{v} \in \mathcal{M}^{k}\left(\left(x_{i}^{-}\right)_{i \in I^{C}},\left(x_{i}^{+}\right)_{i \in I^{C}} ; \underline{L}^{I, \underline{H}, \underline{x}^{ \pm}}, \underline{J}^{I}\right)
$$

with

$$
\begin{aligned}
v_{j j^{\prime}}\left(s_{l}\right):= & \left(v_{j}\left(s_{l}, \delta_{j}\right), \mathcal{P}_{j j^{\prime}}\left(v_{j}\left(s_{l}, \delta_{j}\right), v_{j^{\prime}}\left(s_{l}, 0\right)\right), v_{j^{\prime}}\left(s_{l}, 0\right)\right) \in \mathcal{V}_{\underline{q}}, \\
& G_{n}\left(v_{j j^{\prime}}\left(s_{l}\right)\right)=0 \text { and } d G_{n}\left(v_{j j^{\prime}}\left(s_{l}\right)\right) \neq 0
\end{aligned}
$$

for $0 \leq l \leq k$.

Let $\mathcal{B}$ be the Banach manifold in Equation (7) as in the definition of $\mathcal{M}^{k}\left(\left(x_{i}^{-}\right)_{i \in I^{C}},\left(x_{i}^{+}\right)_{i \in I^{C}} ; \underline{L}^{I, \underline{H}, \underline{x}^{ \pm}}, \underline{J}^{I}\right)$ and recall from Theorem 2.9(b) the transversality of the function

$$
G_{\underline{q}, n}^{\underline{H}, \underline{x}^{ \pm}}: \mathcal{V}_{\underline{\underline{q}, n}, \underline{\underline{x}}^{ \pm}} \rightarrow \mathbb{R}, \quad\left(z_{j}, z_{j^{\prime}}\right) \mapsto G_{n}\left(z_{j}, \mathcal{P}_{j j^{\prime}}\left(z_{j}, z_{j^{\prime}}\right), z_{j^{\prime}}\right)
$$

on the open set

$$
\begin{aligned}
\mathcal{V}_{\underline{\underline{q}, n}, \underline{x}^{ \pm}}:=\left\{\left(z_{j}, z_{j^{\prime}}\right) \in L\right. & L_{j j^{\prime}} \underline{\underline{x}}^{ \pm} \mid \\
& \left.\left(z_{j}, \mathcal{P}_{j j^{\prime}}\left(z_{j}, z_{j^{\prime}}\right), z_{j^{\prime}}\right) \in \mathcal{V}_{\underline{q}}, d G_{n}\left(z_{j}, \mathcal{P}_{j j^{\prime}}\left(z_{j}, z_{j^{\prime}}\right), z_{j^{\prime}}\right) \neq 0\right\} .
\end{aligned}
$$

Then $\mathcal{B}^{\prime \prime}:=\left\{\underline{v} \in \mathcal{B} \mid\left(v_{j}\left(s_{l}, \delta_{j}\right), v_{j^{\prime}}\left(s_{l}, 0\right)\right) \in \mathcal{V}_{\underline{q}, n}^{H, x} \underline{x}^{ \pm} \forall 0 \leq l \leq k\right\}$ is an open subset of $\mathcal{B}$ and

$$
s(\underline{J}, \underline{v}):=\left(\bar{\partial}_{\underline{J}^{I}}(\underline{v}),\left(G_{\underline{q}, n}^{H, \underline{x}^{ \pm}}\left(v_{j}\left(s_{l}, \delta_{j}\right), v_{j^{\prime}}\left(s_{l}, 0\right)\right)_{l=0, \ldots, k}\right)\right.
$$

defines a $\mathcal{C}^{\ell}$ section of the bundle $\left.\mathcal{E}\right|_{\mathcal{B}^{\prime \prime}} \times \mathbb{R}^{k+1} \rightarrow \mathcal{J}_{t}^{\ell}(\underline{\delta}) \times \mathcal{B}^{\prime \prime}$. Its linearized operator at a zero maps $\left(\underline{K}=\left(K_{i}\right)_{i=0, \ldots, r}, \underline{\xi}=\left(\xi_{i}\right)_{i \in I^{C}}\right)$ to

$$
\begin{aligned}
\left(\left(D_{\underline{v}} \bar{\partial}^{I} \underline{I}^{I}\right) \underline{\xi}-\left(K_{i} J_{i} \partial_{s} v_{i}\right)_{i \in I^{C}},\right. & \\
& \left(D_{\left(v_{j}\left(s_{l}, \delta_{j}\right), v_{j^{\prime}}\left(s_{l}, 0\right)\right)} G_{\underline{q}, n^{\underline{H}}, \underline{x}^{ \pm}}\left(\xi_{j}\left(s_{l}, \delta_{j}\right), \xi_{j^{\prime}}\left(s_{l}, 0\right)\right)_{l=0, \ldots, k}\right) .
\end{aligned}
$$

As before, the second summand in the first component is surjective using just the freedom in $K$. The second component is surjective since by Theorem 2.9(b) each map $D_{\left(v_{j}\left(s_{l}, \delta_{j}\right), v_{j^{\prime}}\left(s_{l}, 0\right)\right)} G_{\underline{q}, n^{\underline{H}}, \underline{x}^{ \pm}}$is surjective, and $\underline{\xi}$ can be chosen to assume any given tuple of values on the linearized Lagrangian correspondence $T L \underline{H} \underline{j} j^{\prime} \underline{x}^{ \pm}$at distinct $s_{0}, \ldots, s_{k+1} \in \mathbb{R}$. So by the implicit function theorem $\{(\underline{J}, \underline{v}) \mid s(\underline{J}, \underline{v})=0\}$ is a $\mathcal{C}^{\ell}$ Banach manifold and its projection to $\mathcal{J}_{t}^{\ell}(\underline{\delta})$ is a Fredholm map of class $\mathcal{C}^{\ell}$ and negative index Ind $D_{\underline{v}}-(k+1)=-1$. As before, by the Sard-Smale theorem, the set of regular values is comeagre, and for each regular $\underline{J}$ the set of solutions is empty. This proves the Lemma. 
We now obtain a comeagre subset $\mathcal{J}_{3, j, j^{\prime}}^{\ell} \subset \mathcal{J}_{t}^{\ell}(\underline{\delta})$ by taking the countable intersection of the comeagre sets $\mathcal{J}\left(\underline{q}, n, s_{0}, \ldots, s_{k}\right)$ given by the Lemma with $\mathcal{J}_{2, j, j^{\prime}}^{\ell}$. Now consider any $\underline{J} \in \mathcal{J}_{3, j, j^{\prime}}^{\ell}$ and $\underline{u} \in \bigcup_{k \leq k_{0}} \mathcal{M}^{k}\left(\underline{x}^{-}, \underline{x}^{+} ; \underline{L}, \underline{J}\right)$ with $u_{j+1}, \ldots, u_{j^{\prime}-1}$ constant. As before, this induces a solution

$$
\underline{v}:=\left(u_{i}\right)_{i \in I^{C}} \in \mathcal{M}^{k}\left(\left(x_{i}^{-}\right)_{i \in I^{C}},\left(x_{i}^{+}\right)_{i \in I^{C}} ; \underline{L}^{\left.I, \underline{H}^{\prime} \underline{x}^{ \pm}, \underline{J}^{I}\right)}\right.
$$

such that

$$
\begin{aligned}
\mathcal{P}_{j j^{\prime}}\left(v_{j}\left(s, \delta_{j}\right), v_{j^{\prime}}(s, 0)\right) & =\left(u_{j+1}(s, 0), u_{j^{\prime}-1}\left(s, \delta_{j^{\prime}-1}\right)\right) \\
& =\left(x_{j+1}(0), x_{j^{\prime}-1}\left(\delta_{j^{\prime}-1}\right)\right)
\end{aligned}
$$

is independent of $s \in \mathbb{R}$. Moreover, by Step 2, we know $D_{\left(v_{j}\left(s_{0}, \delta_{j}\right), v_{j^{\prime}}\left(s_{0}, 0\right)\right)} \mathcal{P}_{j j^{\prime}}=0$ for all $s \in \mathbb{R}$, and hence by Proposition 2.8 the intersection $\Lambda\left(v_{j j^{\prime}}(s)\right)$ at

$$
v_{j j^{\prime}}(s):=\left(v_{j}\left(s, \delta_{j}\right), x_{j+1}(0), x_{j^{\prime}-1}\left(\delta_{j^{\prime}-1}\right), v_{j^{\prime}}(s, 0)\right)
$$

induces a Lagrangian subspace of $T_{x_{j+1}(0)} M_{j+1} \times T_{x_{j^{\prime}-1}\left(\delta_{j^{\prime}-1}\right)} M_{j^{\prime}-1}$ for every $s \in \mathbb{R}$. Suppose by contradiction that it is not constant on any neighborhood of $\sigma \in \mathbb{R}$. Fix $\underline{q} \in S_{j j^{\prime}}$ such that $v_{j j^{\prime}}(s) \in \mathcal{V}_{q}$ for $|s-\sigma|<\epsilon$ sufficiently small, then by Theorem 2.9(a) we have $G_{1}\left(v_{j j^{\prime}}(s)\right)=\cdots=\bar{G}_{N}\left(v_{j j^{\prime}}(s)\right)=0$ for all $|s-\sigma|<\epsilon$, but $d G_{n}\left(v_{j j^{\prime}}\left(\sigma^{\prime}\right)\right) \neq 0$ for some $1 \leq n \leq N$ and $\sigma^{\prime} \in(\sigma-\epsilon, \sigma+\epsilon)$. Since the nonvanishing is an open condition, we may also find $s_{0}<\cdots<s_{k} \in \mathbb{Q} \cap(\sigma-\epsilon, \sigma+\epsilon)$ with $d G_{n}\left(v_{j j^{\prime}}\left(s_{l}\right)\right) \neq 0$, in contradiction to the Lemma.

Step 4 Next we explicitly state Step 3 as a splitting property and deduce surjectivity of part of the linearized operator:

If $\underline{u} \in \mathcal{M}^{k}\left(\underline{x}^{-}, \underline{x}^{+} ; \underline{L}, \underline{J}\right)$ with $u_{i}(s, t)=x_{i}^{ \pm}(t)=: x_{i}(t)$ for $i=j+1, \ldots, j^{\prime}-1$ gives rise to a constant Lagrangian subspace as in Step 3, then the linearized seam conditions

$$
\begin{aligned}
T_{\left(u_{j}\left(s, \delta_{j}\right), u_{j+1}(s, 0)\right)} L_{j(j+1)} & =\Lambda_{j}(s) \times \Lambda_{j+1}, \\
T_{\left(u_{j^{\prime}-1}\left(s, \delta_{j^{\prime}-1}\right), u_{j^{\prime}}(s, 0)\right)} L_{\left(j^{\prime}-1\right) j^{\prime}} & =\Lambda_{j^{\prime}-1} \times \Lambda_{j^{\prime}}(s)
\end{aligned}
$$

split into the Lagrangian subspaces

$$
\begin{aligned}
& \Lambda_{j}(s) \cong \quad T_{\left(u_{j}\left(s, \delta_{j}\right), x_{j+1}(0)\right)} L_{j(j+1)} \cap\left(T_{u_{j}\left(s, \delta_{j}\right)} M_{j} \times\{0\}\right) \hookrightarrow T_{u_{j}\left(s, \delta_{j}\right)} M_{j}, \\
& \Lambda_{j+1} \cong \quad T_{\left(u_{j}\left(s, \delta_{j}\right), x_{j+1}(0)\right)} L_{j(j+1)} \cap\left(\{0\} \times T_{x_{j+1}(0)} M_{j+1}\right) \hookrightarrow T_{x_{j+1}(0)} M_{j+1}, \\
& \Lambda_{j^{\prime}-1} \cong T_{\left(x_{j^{\prime}-1}\left(\delta_{j^{\prime}-1}\right), u_{j^{\prime}}(s, 0)\right)} L_{\left(j^{\prime}-1\right) j^{\prime}} \cap\left(T_{x_{j^{\prime}-1}\left(\delta_{j^{\prime}-1}\right)} M_{j^{\prime}-1} \times\{0\}\right) \\
& \hookrightarrow T_{x_{j^{\prime}}\left(\delta_{j^{\prime}}\right)} M_{j^{\prime}}, \\
& \Lambda_{j^{\prime}}(s) \cong T_{\left(x_{j^{\prime}-1}\left(\delta_{j^{\prime}-1}\right), u_{j^{\prime}}(s, 0)\right)} L_{\left(j^{\prime}-1\right) j^{\prime}} \cap\left(\{0\} \times T_{u_{j^{\prime}}(s, 0)} M_{j^{\prime}}\right) \quad \hookrightarrow T_{u_{j^{\prime}}(s, 0)} M_{j^{\prime}}
\end{aligned}
$$


Moreover, the operator $D_{j j^{\prime}}:=\left(\partial_{s}+J_{i}\left(x_{i}\right) \partial_{t}-J_{i}\left(x_{i}\right) D_{x_{i}} X_{H_{i}}\right)_{i=j+1, \ldots, j^{\prime}-1}$ maps

$$
\left\{\begin{array}{l|l}
\xi \in \bigoplus_{i=j+1}^{j^{\prime}-1} W^{1, p}\left(\mathbb{R} \times\left[0, \delta_{i}\right], x_{i}^{*} T M_{i}\right) & \begin{array}{l}
\left(\xi_{i}\left(s, \delta_{i}\right), \xi_{i+1}(s, 0)\right) \in T L_{i(i+1)} \forall i \\
\xi_{j+1}(s, 0) \in \Lambda_{j+1}, \\
\xi_{j^{\prime}-1}\left(s, \delta_{j^{\prime}-1}\right) \in \Lambda_{j^{\prime}-1}
\end{array}
\end{array}\right\}
$$

onto $\bigoplus_{i=j+1}^{j^{\prime}-1} L^{p}\left(\mathbb{R} \times\left[0, \delta_{i}\right], x_{i}^{*} T M_{i}\right)$.

We can express the operator $D_{j j^{\prime}}=\partial_{s}+A$ in terms of an $s$-independent operator

$$
A=\left(J_{i}\left(x_{i}\right) \partial_{t}-J_{i}\left(x_{i}\right) D_{x_{i}} X_{H_{i}}\right)_{i=j+1, \ldots, j^{\prime}-1},
$$

which is self-adjoint on $\bigoplus_{i=j+1}^{j^{\prime}-1} L^{2}\left(\left[0, \delta_{i}\right], x_{i}^{*} T M_{i}\right)$ with domain

$$
\left\{\begin{array}{l|l}
\underline{\zeta} \in \bigoplus_{i=j+1}^{j^{\prime}-1} W^{1,2}\left(\left[0, \delta_{i}\right], x_{i}^{*} T M_{i}\right) & \begin{array}{l}
\left(\zeta_{i}\left(\delta_{i}\right), \zeta_{i+1}(0)\right) \in T_{\left(x_{i}\left(\delta_{i}\right), x_{i+1}(0)\right)} L_{i(i+1)} \forall i \\
\zeta_{j+1}(0) \in \Lambda_{j+1}, \\
\zeta_{j^{\prime}-1}\left(\delta_{j^{\prime}-1}\right) \in \Lambda_{j^{\prime}-1}
\end{array}
\end{array}\right\} .
$$

Moreover, the nondegeneracy of the intersection points $\mathcal{I}(\underline{L}, \underline{H})$ implies that $A$ is invertible. Indeed, the linearized operator cutting out $\mathcal{I}(\underline{L}, \underline{H})$ as trajectory space splits at $\underline{x}^{ \pm}$into $A$ and $\left(J_{i}\left(x_{i}^{ \pm}\right) \partial_{t}-J_{i}\left(x_{i}^{ \pm}\right) D_{x_{i}^{ \pm}} X_{H_{i}}\right)_{i \in\left\{j+1, \ldots, j^{\prime}-1\right\}^{C}}$ with the analogous linearized seam conditions. Now a general spectral analysis and Sobolev embedding argument proves that $D_{j j^{\prime}}$ is in fact an isomorphism. This is the same analysis as outlined at the beginning of the proof, using the elliptic estimates for the quilted Cauchy-Riemann operator in the general proof method given by eg [8, Lemma 2.4; 1, Chapter 3].

Step 5 We deduce from the previous steps that the set of almost complex structures of class $\mathcal{C}^{\ell}$, for which the linearized operators are surjective, is dense in the following sense:

For every integer $\ell>k_{0}$ let $\mathcal{J}_{\text {reg }}^{\ell}$ be the set of $\underline{J} \in \mathcal{J}_{t}^{\ell}(\underline{\delta})$ for which the linearized operators $D_{\underline{u}} \bar{\partial}_{\underline{J}}$ are surjective at all $\underline{u} \in \bigcup_{k \leq k_{0}} \mathcal{M}^{k}\left(\underline{x}^{-}, \underline{x}^{+} ; \underline{L}, \underline{J}\right)$. Then $\mathcal{J}_{\text {reg }}^{\ell} \subset$ $\mathcal{J}_{t}^{\ell}(\underline{\delta})$ is dense.

The density will follow from proving that $\mathcal{J}_{\text {reg }}^{\ell}$ contains the intersection of $\mathcal{J}_{1}^{\ell}$, all $\mathcal{J}_{1, I}^{\ell}$, and all $\mathcal{J}_{3, j, j^{\prime}}^{\ell}$, ie a comeagre and hence dense set. So we need to consider a given

$$
\underline{J} \in \mathcal{J}_{1}^{\ell} \cap \bigcap_{I} \mathcal{J}_{1, I}^{\ell} \cap \bigcap_{j, j^{\prime}} \mathcal{J}_{3, j, j^{\prime}}^{\ell}
$$

and show surjectivity of $D_{\underline{u}} \bar{\partial}_{\underline{J}}$ for all solutions $\underline{u}$.

Step 1.a ensures surjectivity if $\underline{u}$ has no constant components, so it remains to consider $\underline{u} \in \bigcup_{k \leq k_{0}} \mathcal{M}^{k}\left(\underline{x}^{-}, \underline{x}^{+} ; \underline{L}, \underline{J}\right)$ with $\partial_{s} u_{i} \equiv 0 \Leftrightarrow i \in I$ for some subset $I \subset\{0, \ldots, r\}$. 
If all components are constant, then surjectivity follows as in Step 4 from the fact that $D_{\underline{u}} \bar{\partial}_{\underline{J}}=\partial_{S}+A$ is given by the $s$-independent self-adjoint operator

$$
A=\left(J_{i}\left(x_{i}\right) \partial_{t}-J_{i}\left(x_{i}\right) D_{x_{i}} X_{H_{i}}\right)_{i=0, \ldots, r}
$$

with constant Lagrangian seam conditions.

If $\underline{u}$ is a solution with constant components for a proper subset $I \subset\{0, \ldots, r\}$, then as before this induces a solution

$$
\left(u_{j}\right)_{j \in I^{C}} \in \mathcal{M}^{k}\left(\left(x_{j}^{-}\right)_{j \in I^{C}},\left(x_{j}^{+}\right)_{j \in I^{C}} ; \underline{L}^{I, \underline{H}, \underline{x}^{ \pm}}, \underline{J}^{I}\right)
$$

for the locally composed cyclic Lagrangian correspondence $\underline{L}^{I, \underline{H}, \underline{x}^{ \pm}}$consisting of

$$
\begin{cases}L_{j(j+1)} & \text { for } j, j+1 \in I^{C}, \\ L_{j j^{\prime}}^{\underline{H}, \underline{x}^{ \pm}} \subset M_{j} \times M_{j^{\prime}} & \text { for each pair of consecutive indices } j \triangleleft j^{\prime} \in I^{C}, \\ & \text { ie with } j+1, \ldots, j^{\prime}-1 \in I .\end{cases}
$$

Moreover, Step 3 implies that the linearized seam conditions at each consecutive $j \triangleleft j^{\prime} \in I^{C}$ split as in Step 4. As a direct consequence, the locally composed correspondence also splits:

$$
T_{\left(u_{j}\left(s, \delta_{j}\right), u_{j^{\prime}}(s, 0)\right)} L_{j j^{\prime}}^{\underline{H}, \underline{x}^{ \pm}}=\Lambda_{j}(s) \times \Lambda_{j^{\prime}}(s) .
$$

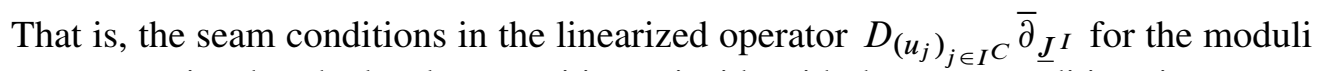
space associated to the local composition coincide with the seam conditions in nonconstant components of the linearized operator $D_{\underline{u}} \bar{\partial}_{\underline{J}}$. Hence the linearized operator for the full problem $D_{\underline{u}} \bar{\partial}_{\underline{J}}$ is the direct sum of $D_{\left(u_{j}\right)_{j \in I} C} \bar{\partial}_{\underline{J}} I$ and the operators $D_{j j^{\prime}}$ as in Step 4 for each consecutive $j \triangleleft j^{\prime} \in I^{C}$. The latter are surjective by Step 4, whereas the former is surjective by Step 1.b. This shows that $D_{\underline{u}} \bar{\partial}_{\underline{J}}$ is indeed surjective for all solutions $\underline{u}$ of index up to $k_{0}$, and hence $\underline{J} \in \mathcal{J}_{\text {reg }}^{\ell}$.

Step 6 As final step we use an intersection argument due to Taubes to transfer from $\mathcal{C}^{\ell}$ to $\mathcal{C}^{\infty}$ almost complex structures. For fixed $\underline{x}^{ \pm} \in \mathcal{I}(\underline{L}, \underline{H}), k_{0} \in \mathbb{N}$ this proves the following:

Let $\mathcal{J}_{\text {reg, } k_{0}}\left(\underline{x}^{-}, \underline{x}^{+}\right)$be the set of $\underline{J} \in \mathcal{J}_{t}(\underline{\delta})$ for which the linearized operators $D_{\underline{u}} \bar{\partial}_{J}$ are surjective for all $\underline{u} \in \bigcup_{k \leq k_{0}} \overline{\mathcal{M}}^{k}\left(\underline{x}^{-}, \underline{x}^{+} ; \underline{L}, \underline{J}\right)$. Then $\mathcal{J}_{\text {reg, } k_{0}}\left(\underline{x}^{-}, \underline{x}^{+}\right) \subset \mathcal{J}_{t}(\underline{\delta})$ is comeagre.

For every $R \geq 0$ let $\mathcal{J}_{\text {reg }}^{R} \subset \mathcal{J}_{t}(\underline{\delta})$ and $\mathcal{J}_{\text {reg }}^{\ell, R} \subset \mathcal{J}_{t}^{\ell}(\underline{\delta})$ for $\ell>k_{0}$ be the sets of $\underline{J}$ for which the linearized operators $D_{\underline{u}} \bar{\partial} \underline{J}$ are surjective at all $\underline{u} \in \bigcup_{k \leq k_{0}} \mathcal{M}^{k}\left(\underline{x}^{-}, \underline{x}^{+} ; \underline{L}, \underline{J}\right)$ with $\left\|\partial_{s} \underline{u}\right\|_{\infty}:=\max _{i}\left\|\partial_{s} u_{i}\right\|_{\infty} \leq R$. 
Then $\mathcal{J}_{\text {reg }}^{R}$ and $\mathcal{J}_{\text {reg }}^{\ell, R}$ are open in the $\mathcal{C}^{\infty}$-, resp. $\mathcal{C}^{\ell}$-topology, by the following compactness and gluing argument as in [3]: Suppose by contradiction that $\underline{J}^{\nu} \rightarrow \underline{J}^{\infty} \in \mathcal{J}_{\text {reg }}^{\ell, R}$ in the $\mathcal{C}^{1}$-topology but $D_{\underline{u}^{\nu}} \bar{\partial}_{\underline{J}^{v}}$ fails to be surjective for some solutions $\bar{\partial}_{\underline{J}}^{v} \underline{u}^{v}=0$ with $\left\|\partial_{s} \underline{u}^{v}\right\|_{\infty} \leq R$. Then a subsequence of $\underline{u}^{v}$ converges to a broken trajectory, consisting of a finite number of nonconstant solutions with respect to $\underline{J}^{\infty}$, and satisfying the same uniform derivative bound. These components cannot have negative index since $\underline{J}^{\infty}$ is regular for indices up to $k_{0}$. So, by index additivity, all components of the broken trajectory have index at most $k_{0}$, and thus the linearized operators at these solutions are surjective. Now a standard gluing construction shows that in fact $D_{\underline{u}^{\nu}} \bar{\partial}_{\underline{J}}{ }^{v}$ must be surjective for some large $v$.

Moreover, $\mathcal{J}_{\text {reg }}^{\ell, R} \subset \mathcal{J}_{t}^{\ell}(\underline{\delta})$ is dense since it contains the dense set $\mathcal{J}_{\text {reg }}^{\ell}$ from Step 5. Now $\mathcal{J}_{\text {reg }}^{R} \subset \mathcal{J}_{t}(\underline{\delta})$ is dense in the $\mathcal{C}^{\infty}$-topology since $\mathcal{J}_{\text {reg }}^{R}=\mathcal{J}_{\text {reg }}^{\ell, R} \cap \mathcal{J}_{t}(\underline{\delta})$, where $\mathcal{J}_{\text {reg }}^{\ell, R} \subset$ $\mathcal{J}_{t}^{\ell}(\underline{\delta})$ is open and dense in the $\mathcal{C}^{\ell}$-topology for all $\ell>k_{0}$. Finally $\mathcal{J}_{\text {reg, }} k_{0}\left(\underline{x}^{-}, \underline{x}^{+}\right)=$ $\bigcap_{R \in \mathbb{N}} \mathcal{J}_{\text {reg }}^{R}$ is a countable intersection of open dense subsets, ie comeagre.

To finish the proof of the Theorem, let $\mathcal{J}_{\text {reg }}(\underline{L} ; \underline{H})$ be the set of $\underline{J} \in \mathcal{J}_{t}(\underline{\delta})$ for which the linearized operators $D_{\underline{u}} \bar{\partial}_{J}$ are surjective for all $\underline{u} \in \mathcal{M}^{k}\left(\underline{x}^{-}, \underline{x}^{+} ; \underline{L}, \underline{J}\right)$ with $\underline{x}^{ \pm} \in \mathcal{I}(\underline{L}, \underline{H})$ and $k \in \mathbb{Z}$. Then $\mathcal{J}_{\text {reg }}(\underline{L} ; \underline{H})=\bigcap_{k_{0} \in \mathbb{N}} \bigcap_{\underline{x}^{ \pm} \in \mathcal{I}(\underline{L}, \underline{H})} \mathcal{J}_{\text {reg, } k_{0}}\left(\underline{x}^{-}, \underline{x}^{+}\right)$ is comeagre in $\mathcal{J}_{t}(\underline{\delta})$ since it is the countable intersection of comeagre sets.

\section{References}

[1] S K Donaldson, Floer homology groups in Yang-Mills theory, Cambridge Tracts in Math. 147, Cambridge Univ. Press (2002) MR1883043 With the assistance of M Furuta and D Kotschick

[2] A Floer, The unregularized gradient flow of the symplectic action, Comm. Pure Appl. Math. 41 (1988) 775-813 MR948771

[3] A Floer, H Hofer, D Salamon, Transversality in elliptic Morse theory for the symplectic action, Duke Math. J. 80 (1995) 251-292 MR1360618

[4] D McDuff, D Salamon, J-holomorphic curves and symplectic topology, Amer. Math. Soc. Colloquium Publ. 52, Amer. Math. Soc. (2004) MR2045629

[5] T Perutz, A symplectic Gysin sequence arXiv:0807.1863

[6] J Robbin, D Salamon, The spectral flow and the Maslov index, Bull. London Math. Soc. 27 (1995) 1-33 MR1331677

[7] H L Royden, Real analysis, third edition, Macmillan, New York (1988) MR1013117

[8] D Salamon, Lectures on Floer homology, from: "Symplectic geometry and topology (Park City, UT, 1997)", (Y Eliashberg, L Traynor, editors), IAS/Park City Math. Ser. 7, Amer. Math. Soc. (1999) 143-229 MR1702944 
[9] K Wehrheim, C T Woodward, Functoriality for Lagrangian correspondences in Floer theory, Quantum Topol. 1 (2010) 129-170 MR2657646

[10] K Wehrheim, C T Woodward, Quilted Floer cohomology, Geom. Topol. 14 (2010) 833-902 MR2602853

[11] K Wehrheim, C T Woodward, Floer cohomology and geometric composition of Lagrangian correspondences, to appear in Adv. Math. arXiv:0905.1368

[12] K Wehrheim, C T Woodward, Pseudoholomorphic quilts, to appear in J. Symp. Geom. arXiv:0905.1369

[13] K Wehrheim, C T Woodward, Exact triangles for fibered Dehn twists, in preparation

[14] K Wehrheim, C T Woodward, Floer field theory, in preparation

Department of Mathematics, Massachusetts Institute of Technology

Cambridge, MA 02139, USA

Department of Mathematics, Rutgers University

Piscataway, NJ 08854, USA

katrin@math.mit.edu, ctw@math.rutgers.edu

www-math.mit.edu/ katrin, www.math.rutgers.edu/ ctw

Received: 2 February 2011 Revised: 11 October 2011 